\title{
Minéralogie de l'indice plombifère de Petite-Hoursinne (Érezée, Province de Luxembourg, Belgique)
}

\author{
Michel BlONDIEAU ${ }^{1}$, Stéphane PUCCIO ${ }^{2}$ et Frédéric HATERT ${ }^{3}$ \\ Manuscrit reçu le 14 mars 2021 et accepté le 1er juin 2021 \\ ${ }^{3}$ Université de Liège, Laboratoire de Minéralogie B18, B-4000 Liège, Belgique
}

${ }^{1}$ Val des Cloches 131, B-6927 Tellin, Belgique

${ }^{2}$ Rue des Fontaines 156, B-4041 Vottem, Belgique

\section{Résumé}

L'observation d'échantillons récoltés dans les déblais d'une petite galerie de recherches minières à Petite-Hoursinne (Erezée, Province de Luxembourg, Belgique) a révélé la présence de deux arséniates : l'érythrite et la mimétite. Ces deux espèces sont peu fréquentes en Belgique et ont déjà été signalées notamment à La Roche-en-Ardenne, une ancienne mine de plomb localisée dans la même formation géologique : la formation de Villé (Praguien, Dévonien inférieur). Cette formation contient des niveaux carbonatés et est caractérisée par la présence d'un peu de plomb qui, dans les zones faillées, forme des petits filons à gangue quartzeuse (voir annexe). La présence d'arséniates dans ces roches ardennaises est surprenante et justifie à elle seule cette note.

Mots clés : érythrite, mimétite, Petite-Hoursinne, formation de Villé, Belgique

\begin{abstract}
Erythrite and mimetite, two arsenates rarely found in Belgium, were discovered near a small mine at Petite-Hoursinne, near Erezée (Luxembourg province, Belgium). That rare occurrence of these arsenates is to be compared with the one observed at La-Roche-en-Ardenne in the same geological Formation (Villé formation, Praguian, Lower Devonian).
\end{abstract}

Keywords : erythrite, mimetite, Petite-Hoursinne, Villé formation, Belgium

\section{Introduction}

Une petite galerie de recherches minières, située le long du ruisseau de Hoursinne (coordonnées Lambert 2008 : 740386, 613918), est signalée par Lohest (1900) comme contenant des filons de galène. Voici ce qu'il écrit : "Au nord de Petite-Hoursinne (Harre), entre cette localité et Laid-Loiseau, existent quelques filons de galène, situés dans des cassures du Coblencien. Ces filons, à en juger par les excavations qu'on rencontre dans la région, ont donné lieu jadis à des tentatives d'exploitation ou à des recherches." Lohest (1900) localise erronément ces travaux de Hoursinne (commune d'Erezée) à Harre, un hameau rattaché aujourd'hui à la commune de Manhay. Stainier (1902) situe cette mine très exactement sur la carte géologique (1:40000) qu'il a dressée ( $\mathrm{N}^{\circ} 169$ Durbuy-Mormont) et précise qu'il s'agit d'un indice plombifère. 
Nous n'avons trouvé aucun autre document faisant état de ces recherches minières si ce n'est une carte indiquant les sites métallurgiques en terre de Durbuy au $\mathrm{XVI}^{\mathrm{e}}$ siècle et sur laquelle sont également situées des minières d'où l'on tirait du minerai de fer pour alimenter les forges des environs (Pirotte, 1966). Sur cette carte, des minières sont localisées au nord de PetiteHoursinne notamment, là où se trouve la galerie.

Jean-Marc Marion et Laurent Barchy visitent le site le 22 mars 2007 et le localisent à $920 \mathrm{~m}$ au NE de la Chapelle de Petite-Hoursinne (à l'E de Mormont), au lieu-dit "Trou Périlleux". Ils le décrivent comme étant "l'entrée d'une vieille mine de fer" (Marion \& Barchy, 2007). Initialement, les recherches devaient probablement avoir été réalisées pour le minerai de fer et c'est sans doute au cours de ces travaux que des filonnets de galène ont été observés.

Nous avons retrouvé cette petite galerie appelée aujourd'hui "Trou Périlleux" (Figures 1 et 2) et que Lohest (1900) avait visitée il y a plus d'un siècle. Elle ne se trouve qu'à quelques mètres au-dessus du ruisseau d'Hoursinne et à une distance d'environ 5 mètres de ce dernier. Cette galerie horizontale, ne présentant aucun danger particulier, s'enfonce d'abord d'une dizaine de mètres perpendiculairement à l'axe de la rivière puis tourne à angle droit vers la droite sur encore 17 mètres environ.

Il est encore possible d'observer un puits comblé et une autre fouille à une dizaine de mètres plus haut que l'œil de la galerie près de laquelle ne se trouvent que quelques haldes fort maigres par rapport aux volumes qui ont été extraits et que l'on peut chiffrer à une centaine de $\mathrm{m}^{3}$ d'après les dimensions de la cavité. Les matériaux sortis de la galerie ont-ils été transportés ailleurs? Mais pourquoi ? D'autant que leur transport ne devait pas être commode puisque la galerie est nichée au fond d'une vallée difficile d'accès. Par ailleurs, les observations faites tant dans la galerie que dans les haldes ne nous ont pas permis de voir du minerai vraiment commercialisable. Le minerai de fer observé est une mauvaise limonite pulvérulente dans laquelle ne se trouvent que quelques traces de galène. Toujours est-il que des travaux assez conséquents y ont été réalisés, sans doute dans l'espoir de trouver un bon filon de galène, laquelle avait, au XIX ${ }^{\mathrm{e}}$ siècle, beaucoup de valeur puisqu'on estime qu'une dizaine de kilos seulement couvrait, à cette époque, le salaire journalier d'un ouvrier (Blondieau, 1993).
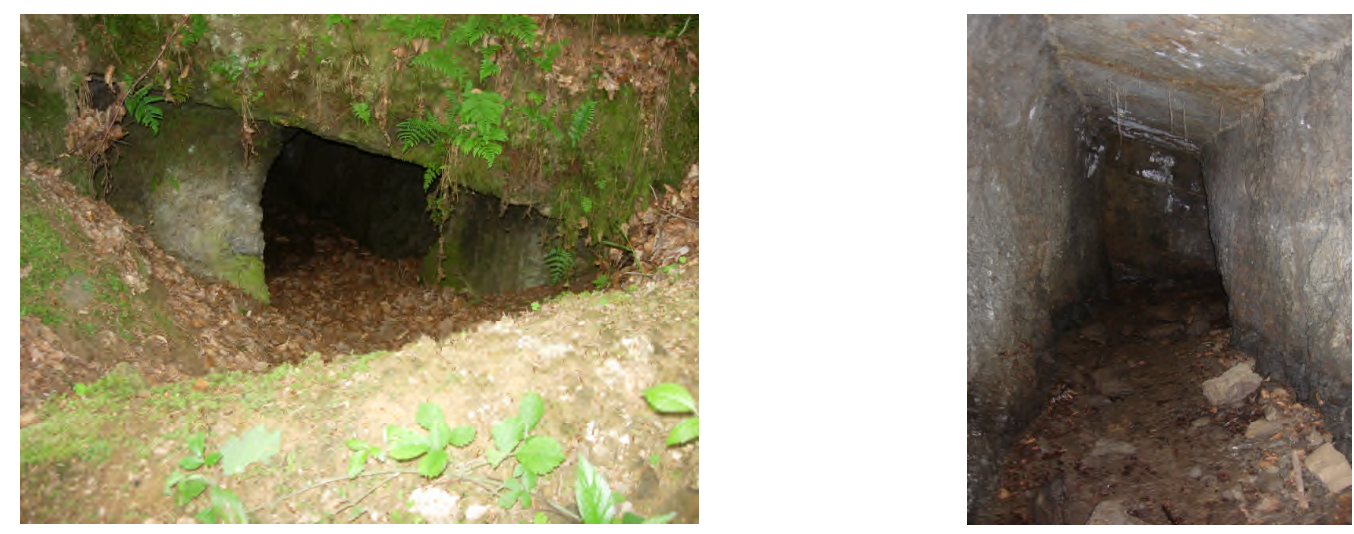

Figure 1 : Eil de la galerie de Petite-Hoursinne et premier tronçon de la galerie. Photos mai 2017. 


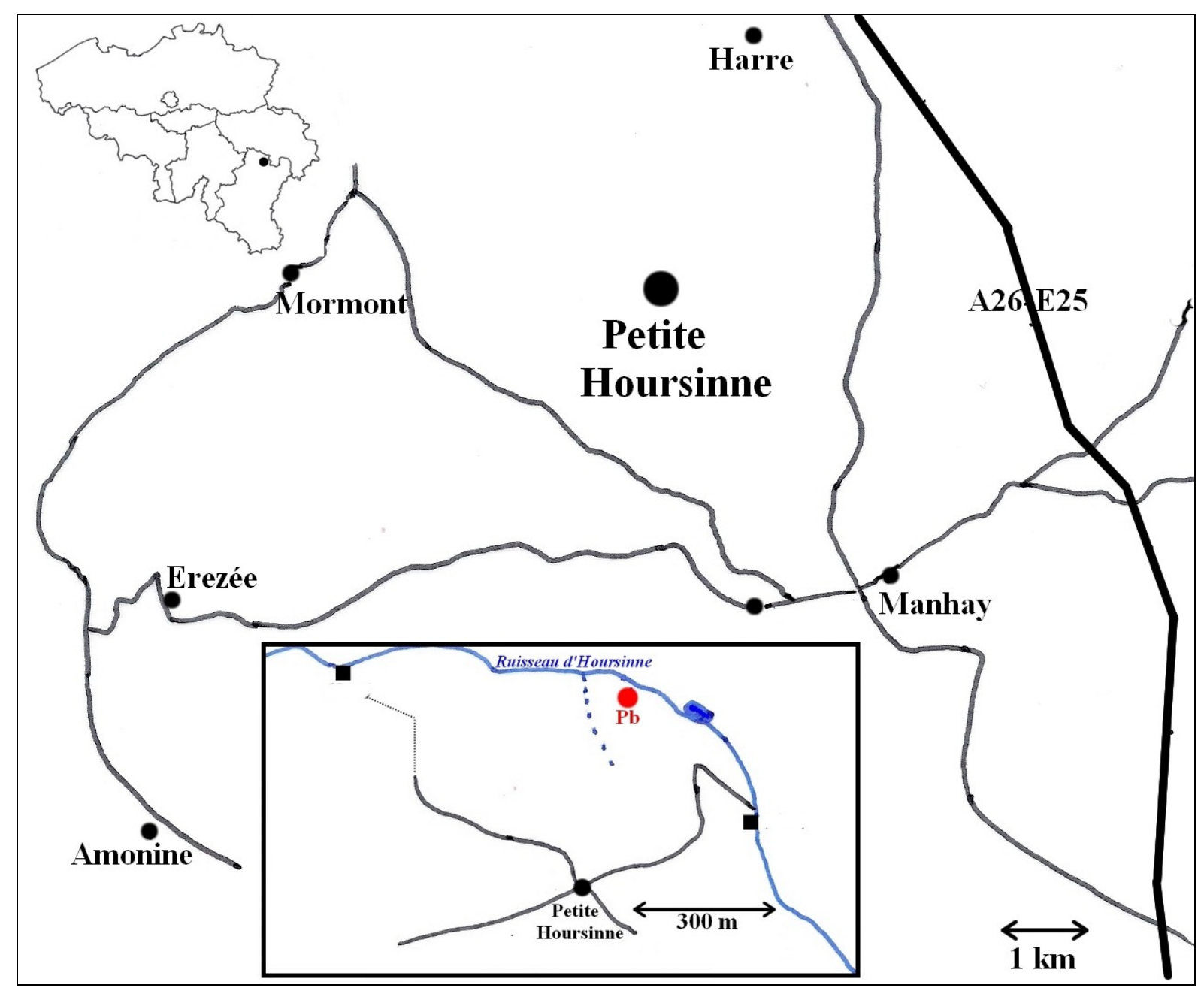

Figure 2 : Localisation de Petite Hoursinne et, en médaillon, localisation de la galerie au lieudit "Trou Périlleux". Les carrés noirs indiquent les sites d'échantillonnage pour analyses géochimiques sur sédiments (Sonnet, 2017).

Le but de cette note est de décrire la minéralogie de ce petit gisement, afin de compléter l'inventaire minéralogique de notre pays.

\section{Contexte géologique}

Marion \& Barchy (2007) indiquent qu'à l'entrée de la galerie, les roches sont des schistes limoniteux et des grès limoniteux appartenant à la formation de Villé (VIL). Cette formation appartient au Praguien moyen (Dévonien inférieur) et se caractérise par la présence de quartzophyllades souvent gréseux, des phyllades parfois quartzeux et des bancs gréseux parfois bleutés particulièrement calcaires et fossilifères. Ces roches présentent souvent une patine superficielle brun rouille.

Sur la carte géologique $\mathrm{N}^{\circ} 169$, Stainier (1902) indique qu'il s'agit de "l'assise inférieure du système Coblencien Cb2a", et Asselberghs (1946) attribue ces roches au faciès des Amonines du Siegenien moyen S2 (Tableau 1). L'abondance de calcaire dans certains bancs est typique 
de cette formation du Dévonien inférieur ardennais, lequel en est pourtant généralement exempt.

La description fournie par Lohest (1900) confirme les caractéristiques de cette formation : "Ayant eu l'occasion de visiter l'une de ces recherches [il s'agit de cette galerie], j'ai pu constater que le filon, très pauvre dans le schiste pur, s'enrichit au contact de certaines roches calcareuses, fossilifères."

Tableau 1: Correspondance approximative de quelques formations régionales et des terminologies anciennes utilisées dans la carte géologique au 1 :40000 (1890-1919) et par Asselberghs (1946). Le trait noir horizontal épais indique la limite entre Dévonien inférieur et Dévonien moyen selon les différentes conceptions.

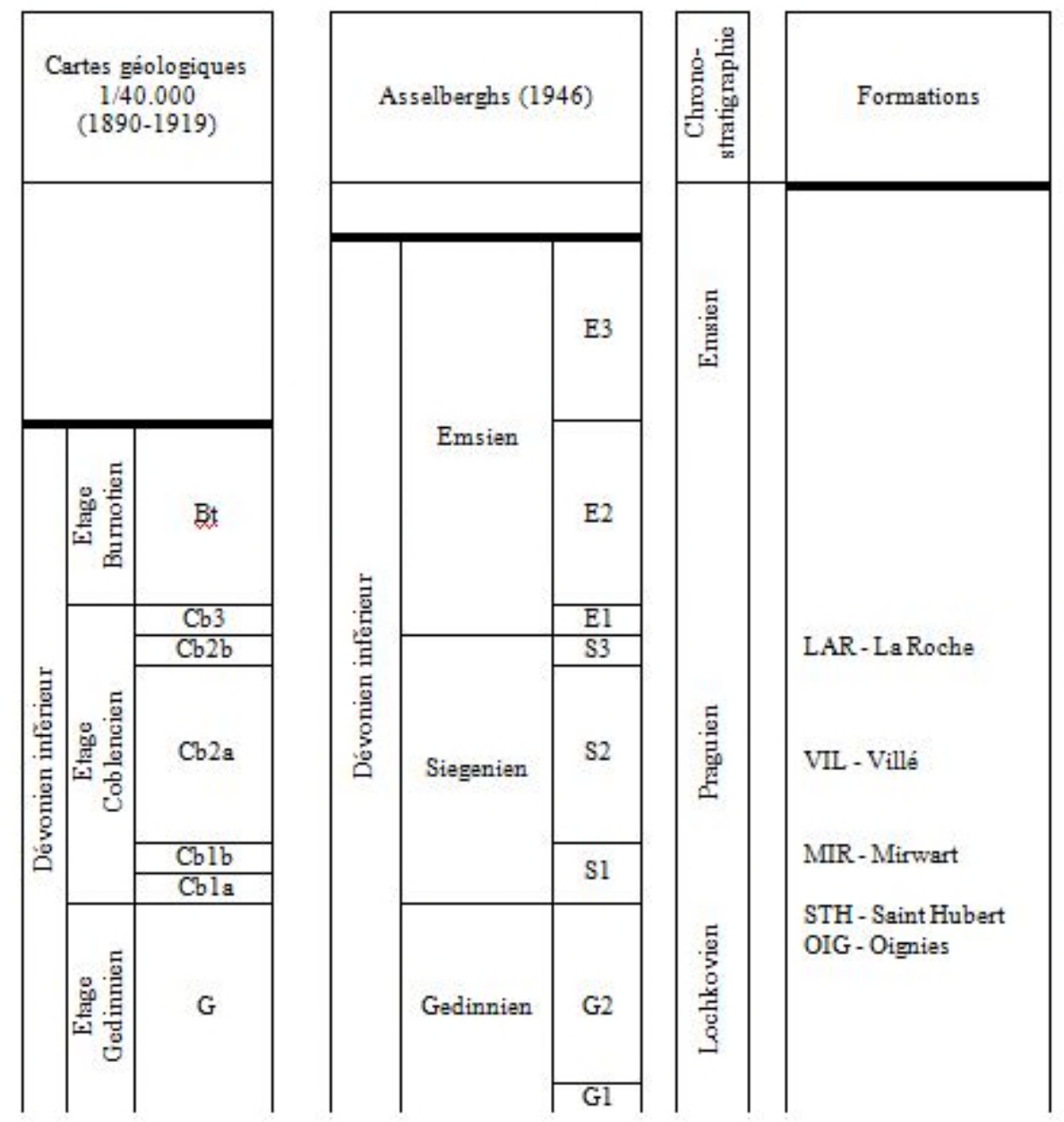

\section{Descriptions des espèces minérales de Petite-Hoursinne}

Les échantillons étudiés ont été prélevés dans la galerie et sur les petites haldes le long du ruisseau d'Hoursinne. Ils ont été examinés sous la loupe binoculaire et l'identité de plusieurs espèces a été confirmée par diffraction des rayons $\mathrm{X}$ sur un diffractomètre de poudres BRUKED D8 ECO utilisant la radiation $\operatorname{CuK}_{\alpha}(\lambda=1,5418 \AA \AA)$. Lorsque les quantités de matière étaient très faibles, un diffractomètre à 4 cercles Rigaku Xcalibur, équipé d'un 
détecteur EOS et utilisant la radiation $\operatorname{MoK}_{\alpha}(\lambda=0,7107 \AA)$, a été utilisé en mode «DebyeScherrer $»$.

La morphologie des minéraux a été observée à l'aide d'un microscope électronique à balayage environnemental FEI ESEM-FEG XL 30 (CAREM-ULg, Cellule d'Appui à la Recherche et à l'Enseignement en Microscopie de l'Université de Liège). Les images ont été obtenues en électrons secondaires (mode «haut-vide») sous une tension d'accélération de $15 \mathrm{kV}$ et sur des échantillons métallisés au platine ou à l'or. Des spectres EDS (« Energy Dispersive X-ray Spectrometry ») de microanalyse élémentaire aux rayons $\mathrm{X}$ ont été obtenus avec le même microscope, équipé d'un détecteur de rayons X Si(Li) EDAX ou SDD Bruker $129 \mathrm{eV}$. Selon les cas, la tension d'accélération était réglée à 15,20 ou $30 \mathrm{kV}$. Les spectres ont été traités soit par le logiciel EDAX, soit par le logiciel Quanta Esprit 1.9 qui a fourni l'identification des éléments.

Nous avons également déterminé la composition chimique de quelques échantillons avec un spectromètre à fluorescence X portable Niton XL3T-980 GOLDD+ de chez Thermo Fisher. Ce spectromètre possède un tube à rayon X Niton XL3T composé d'une anode d'or pouvant émettre un faisceau de $3 \mathrm{~mm}$ de diamètre. Il travaille sous une différence de potentiel de 9 à $50 \mathrm{kV}$, et un courant de 0 à $40 \mu \mathrm{A}$. Les rayons $\mathrm{X}$ réémis passent ensuite dans un détecteur de type GOLDD (Geometrically Optimized Large Area Drift Detector) d'une résolution : $<185$ $\mathrm{eV}$ à $60000 \mathrm{cps}$ pour un temps de mise en forme de $4 \mu \mathrm{s}$.

Les minéraux sont décrits par ordre alphabétique, et leurs formules chimiques idéales sont tirées de la liste officielle de la CNMNC-IMA (Commission on New Minerals, Nomenclature and Classification of the International Mineralogical Association).

\subsection{Anglésite, $\mathrm{PbSO}_{4}$}

L'anglésite est identifiée visuellement en cristaux présentant l'habitus en "burin" assez caractéristique de l'espèce (Figure 3). À Petite-Hoursinne, le minéral n'est pas fréquent.

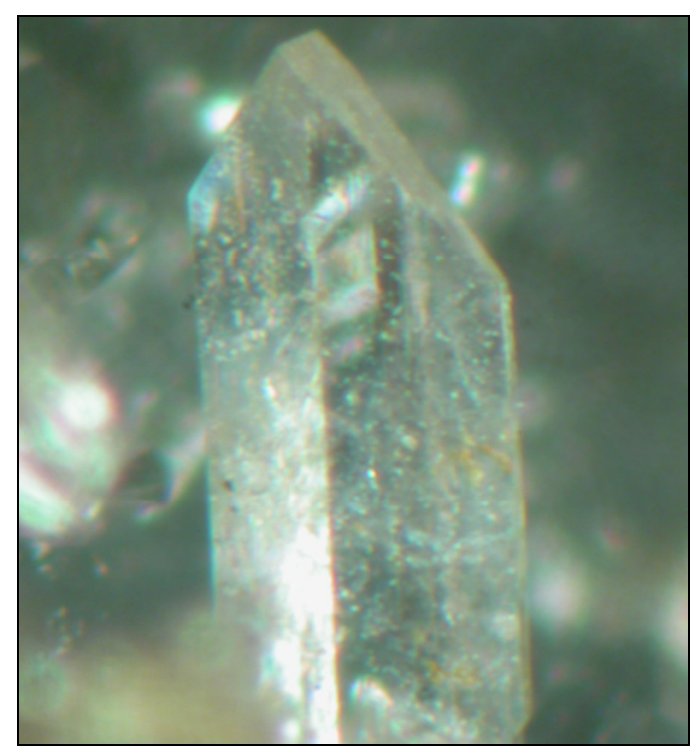

Figure3 : Cristal d'anglésite de Petite-Hoursinne. $\mathrm{L}=2 \mathrm{~mm}$. Photo M. Blondieau 


\subsection{Aragonite, $\mathrm{CaCO}_{3}$}

L'aragonite se présente en aiguilles blanches de quelques millimètres de longueur, dans des cavités de brachiopodes fossiles avec dolomite, calcite, quartz et parfois érythrite (Figure 4). Le minéral est identifié par diffraction des rayons $\mathrm{X}$ ainsi que par des analyses chimiques qualitatives EDS qui mettent bien en évidence la seule présence des éléments $\mathrm{O}, \mathrm{C}$ et $\mathrm{Ca}$.

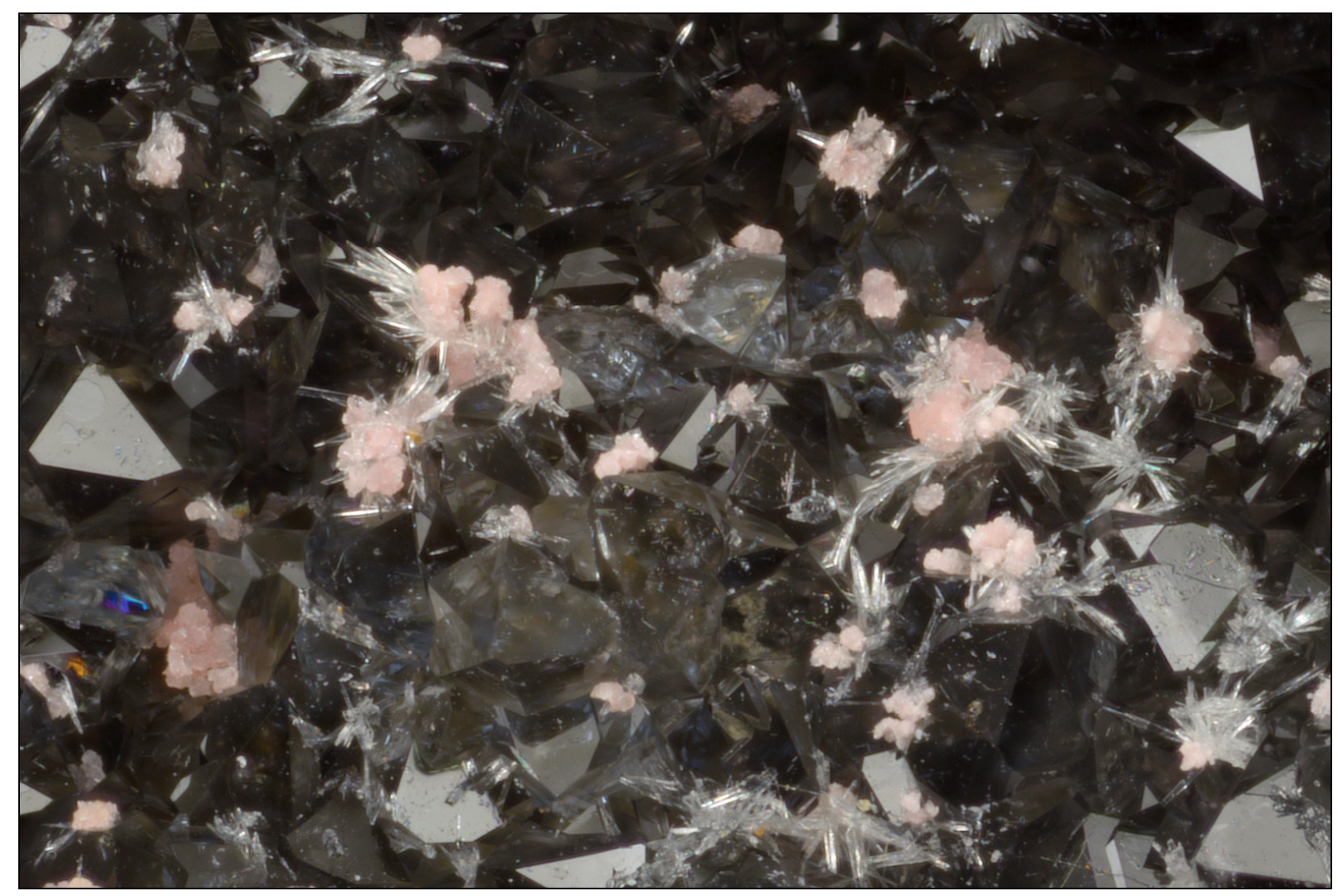

Figure 4 : Aragonite et érythrite sur quartz de Petite-Hoursinne. $\mathrm{L}=1,50 \mathrm{~mm}$. Photo P. Chollet.

\subsection{Calcite, $\mathrm{CaCO}_{3}$}

De petits cristaux associés à la dolomite et à l'aragonite sont visuellement déterminés comme de la calcite, ce qui est confirmé grâce à une analyse par diffraction des rayons X. La calcite semble également former de minuscules cristaux de seconde génération sur la dolomite. Enfin, les clivages rhomboédriques observés sur le minéral constituant des articles de crinoïdes fossiles suggèrent qu'il s'agit également de calcite. La calcite est encore identifiée par diffraction des rayons $\mathrm{X}$ dans des enduits blancs observées sur les parois de la galerie, où elle se trouve en mélange avec de l'aragonite.

\subsection{Cérusite, $\mathrm{PbCO}_{3}$}

La cérusite est identifiée visuellement et par diffraction des rayons $\mathrm{X}$ en petits cristaux sur la galène (Figure 5A). Elle se présente également en beaux cristaux maclés (Figure 5B). 

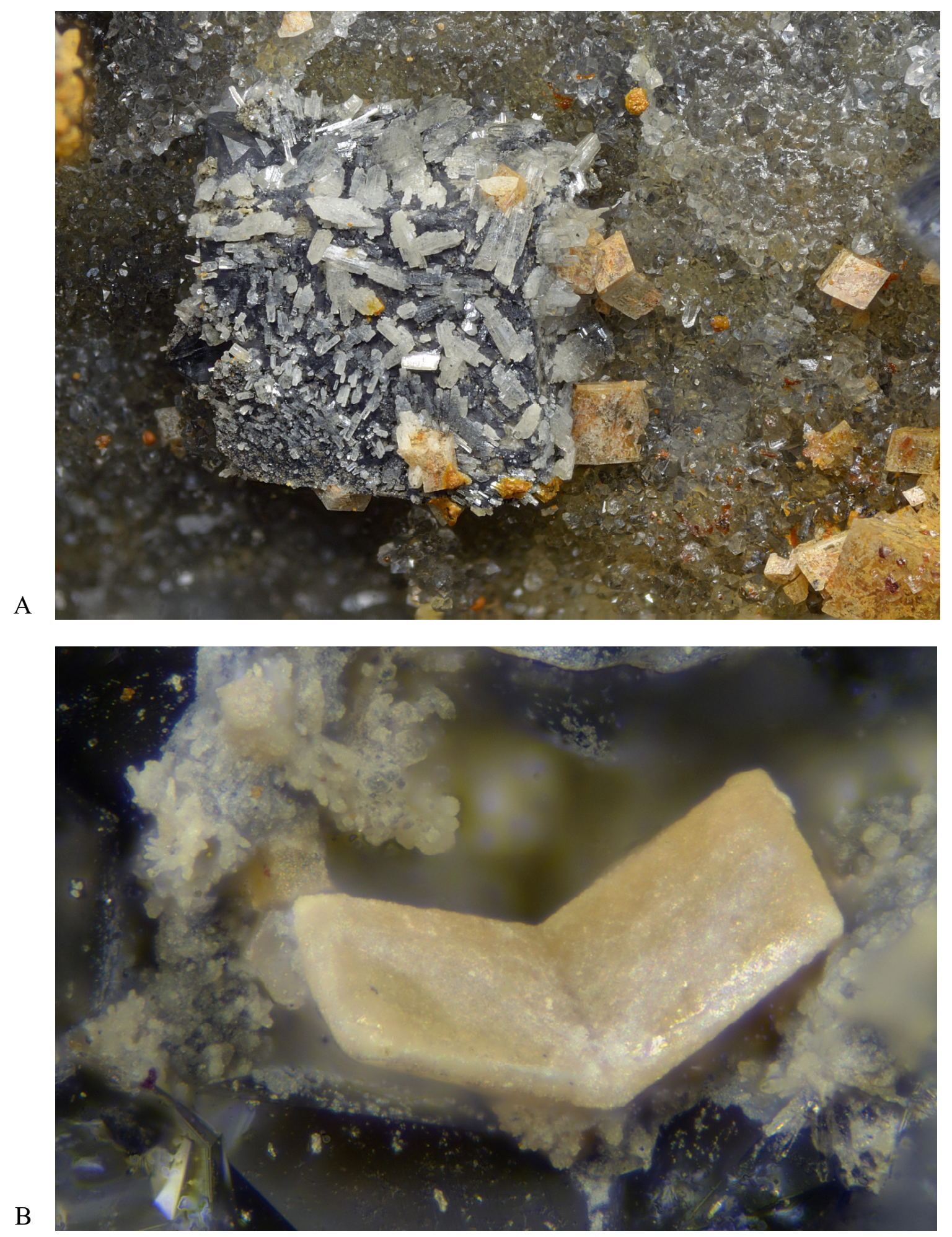

Figure 5 : Cérusite de Petite-Hoursinne. (A) Petits cristaux sur cube de galène très corrodée avec dolomite et quartz. $\mathrm{L}=4,4 \mathrm{~mm}$. (B) Cristaux maclés $\mathrm{L}=0,49 \mathrm{~mm}$.

Photos P. Chollet. 


\subsection{Chalcopyrite, $\mathrm{CuFeS}_{2}$}

La chalcopyrite se présente en mouchetures dans le quartz et en inclusions dans la galène. Son identification visuelle est confirmée par des analyses par diffraction des rayons X.

Ailleurs dans la formation de Villé, la chalcopyrite est généralement discrète mais assez fréquemment observée avec la galène. C'est notamment le cas à La Roche-en-Ardenne (Blondieau \& Hatert, 2009) et à Masbourg où elle est localement un peu plus abondante (Blondieau et al., 2017).

\subsection{Covellite, $\mathrm{CuS}$}

Une auréole bleue violacée autour d'une petite plage millimétrique de chalcopyrite est identifiée visuellement comme de la covelline. Une analyse par diffraction des rayons X met bien en évidence l'association chalcopyrite et covellite.

\subsection{Dolomite, $\mathrm{CaMg}\left(\mathrm{CO}_{3}\right)_{2}$}

La dolomite se montre en jolis cristaux rhomboédriques de plusieurs millimètres de diamètre, presque incolores ou blancs, voire parfois un peu rosés lorsqu'on les découvre peu altérés dans les roches bleutées très compactes (Figure 6). Ce minéral est souvent associé à un peu de galène et plus rarement à l'érythrite. La dolomite se présente également dans les moules internes des gros brachiopodes fossiles qui caractérisent la formation de Villé (Figure 7). Enfin, dans les cavités de la roche à patine brune, la dolomite est moins fraîche et de teinte plus brune, coloration due à des oxydes de fer dont la goethite. L'identification visuelle de la dolomite est confirmée par diffraction des rayons $\mathrm{X}$.

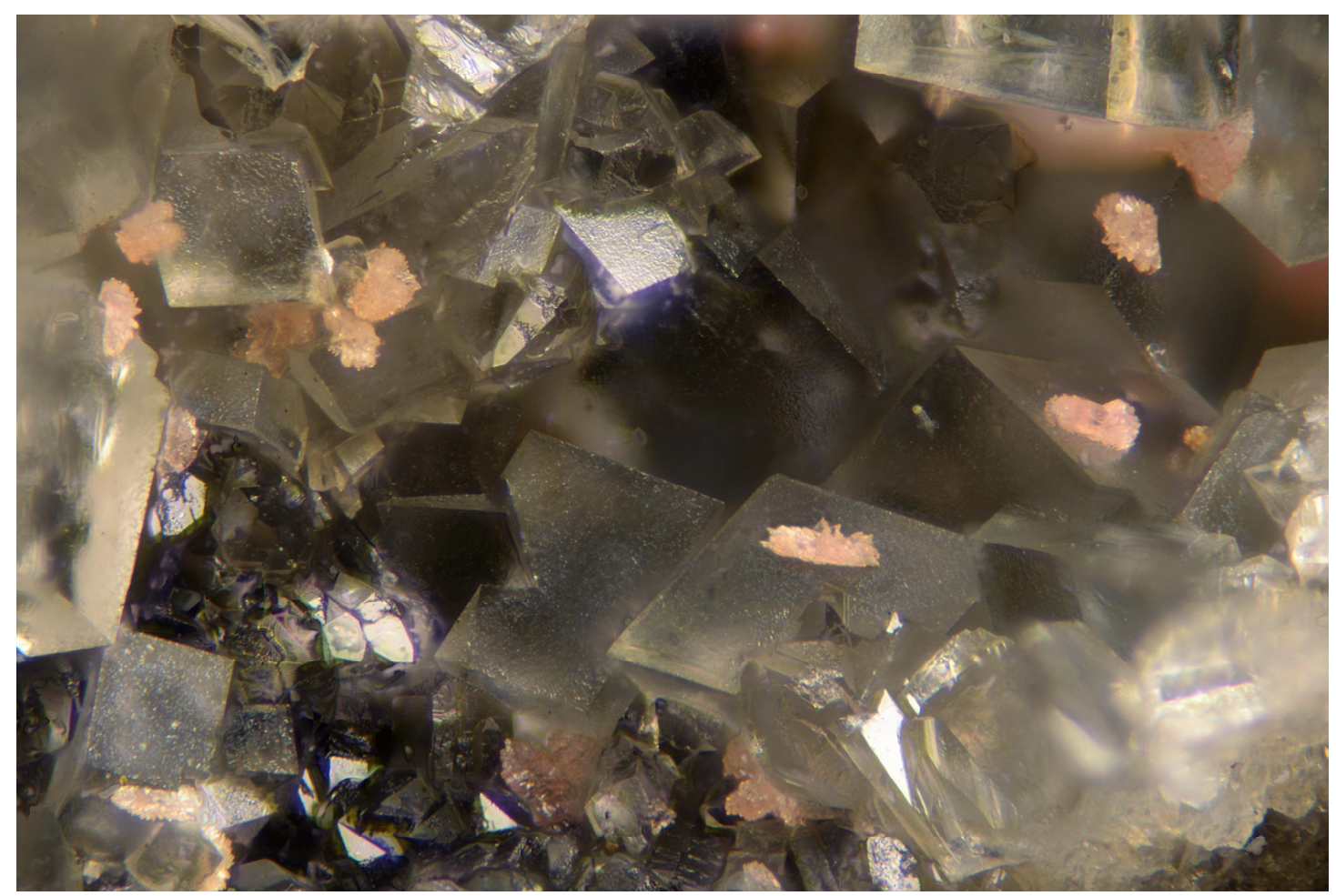

Figure 6 : Dolomite et érythrite de Petite-Hoursinne. $\mathrm{L}=0,86 \mathrm{~mm}$. Photo P. Chollet. 


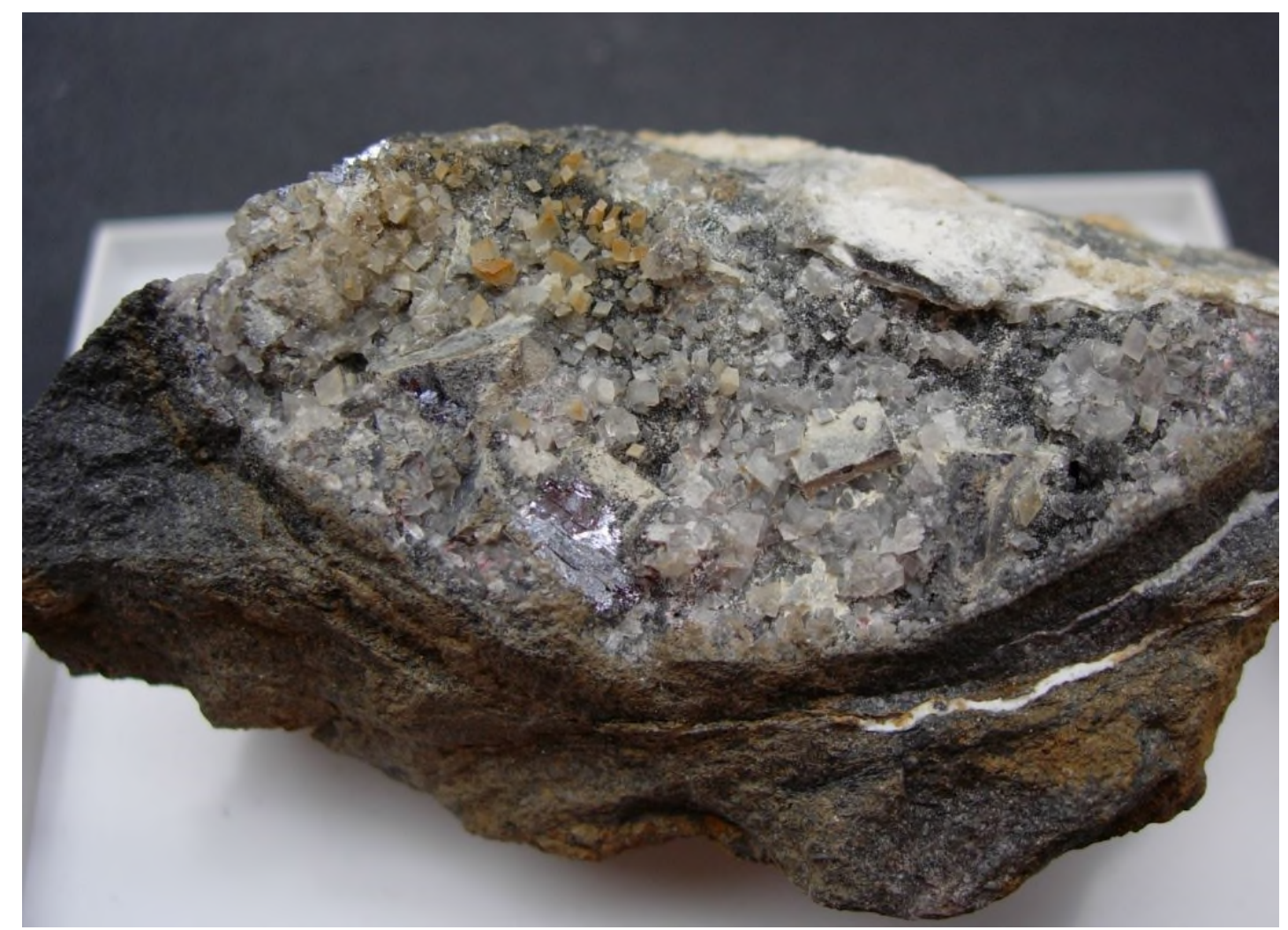

Figure 7 : Dolomite en cristaux rhomboédriques tapissant l'intérieur d'une grande coquille de brachiopode fossile de Petite-Hoursinne. $\mathrm{L}=4 \mathrm{~cm}$. Photo M. Blondieau.

La partie blanche dans la partie inférieure droite est constituée de kaolinite.

\section{8. Érythrite, $\mathrm{Co}_{3}\left(\mathrm{AsO}_{4}\right)_{2.8 \mathrm{H}_{2} \mathrm{O}}$}

Associés à la dolomite non altérée et parfois à la sphalérite, de petits agrégats roses inframillimétriques sont connus depuis longtemps à Petite-Hoursinne (Figures 8 à 11). Ils sont identifiés par diffraction des rayons X comme étant de l'érythrite (arséniate de cobalt) ou de la köttigite (arséniate de zinc). Ces deux espèces forment une solution solide continue, et il est difficile de les différencier par diffraction des rayons $X$.

Une de ces populations, analysée par EDS, montre la présence des seuls éléments majoritaires $\mathrm{Co}$, As et $\mathrm{O}$, ainsi que $\mathrm{Ni}$ dont la teneur est apparemment proche de $40 \%$ de celle du cobalt. Une autre population, qu'il n'est pas possible de différencier visuellement de la première, montre une présence majoritaire des éléments $\mathrm{O}$, As et $\mathrm{Co}$, mais aussi $\mathrm{Zn}$ ainsi que Ni plus minoritaire. L'association à la sphalérite, ainsi que la présence de zinc parfois très importante constatée dans quelques échantillons analysés par EDS, nous ont conduits à suspecter la köttigite, mais nous n'avons cependant pas réussi à trouver un échantillon qui présentait une teneur en zinc supérieure à celle du cobalt. Tous ces échantillons roses de Petite-Hoursinne seront donc assimilés à l'érythrite, le pôle cobaltifère de cette série de minéraux.

En microscopie électronique, l'érythrite de Petite-Hoursinne se présente parfois en beaux mais minuscules agrégats de cristaux. Leur taille est de l'ordre de la dizaine de microns 
seulement (Figure 12). Les photos permettent d'observer la morphologie monoclinique de ces cristaux (Figures 13 et 14).

L'érythrite est un minéral peu fréquent en Belgique qui n'a été rencontré qu'à La Roche-enArdenne (Blondieau \& Hatert, 2009). Petite-Hoursinne est donc la seconde occurrence de ce minéral pour la Belgique.

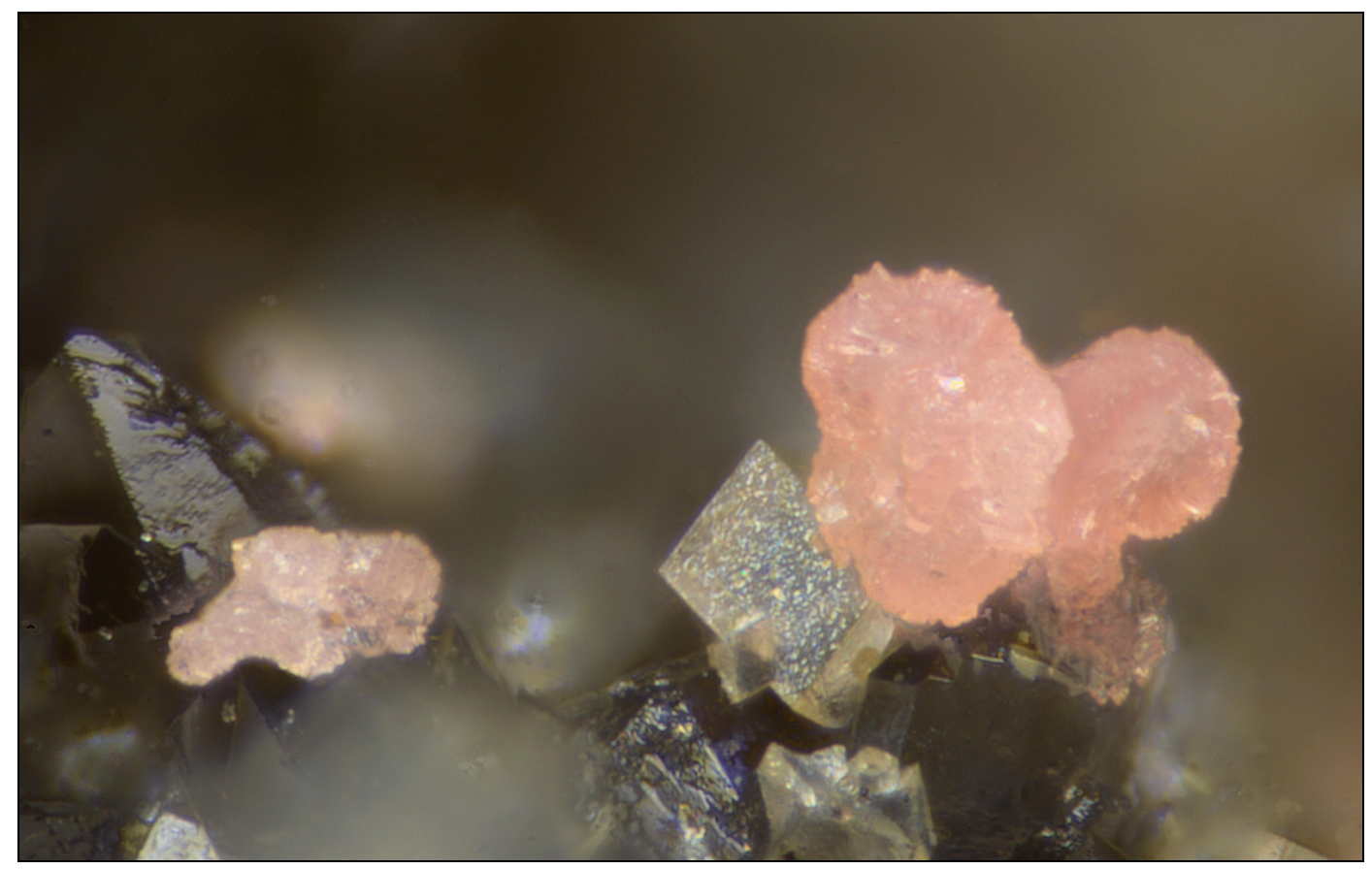

Figure 8 : Érythrite sur dolomite de Petite-Hoursinne. $\mathrm{L}=0,33 \mathrm{~mm}$. Photo P. Chollet.

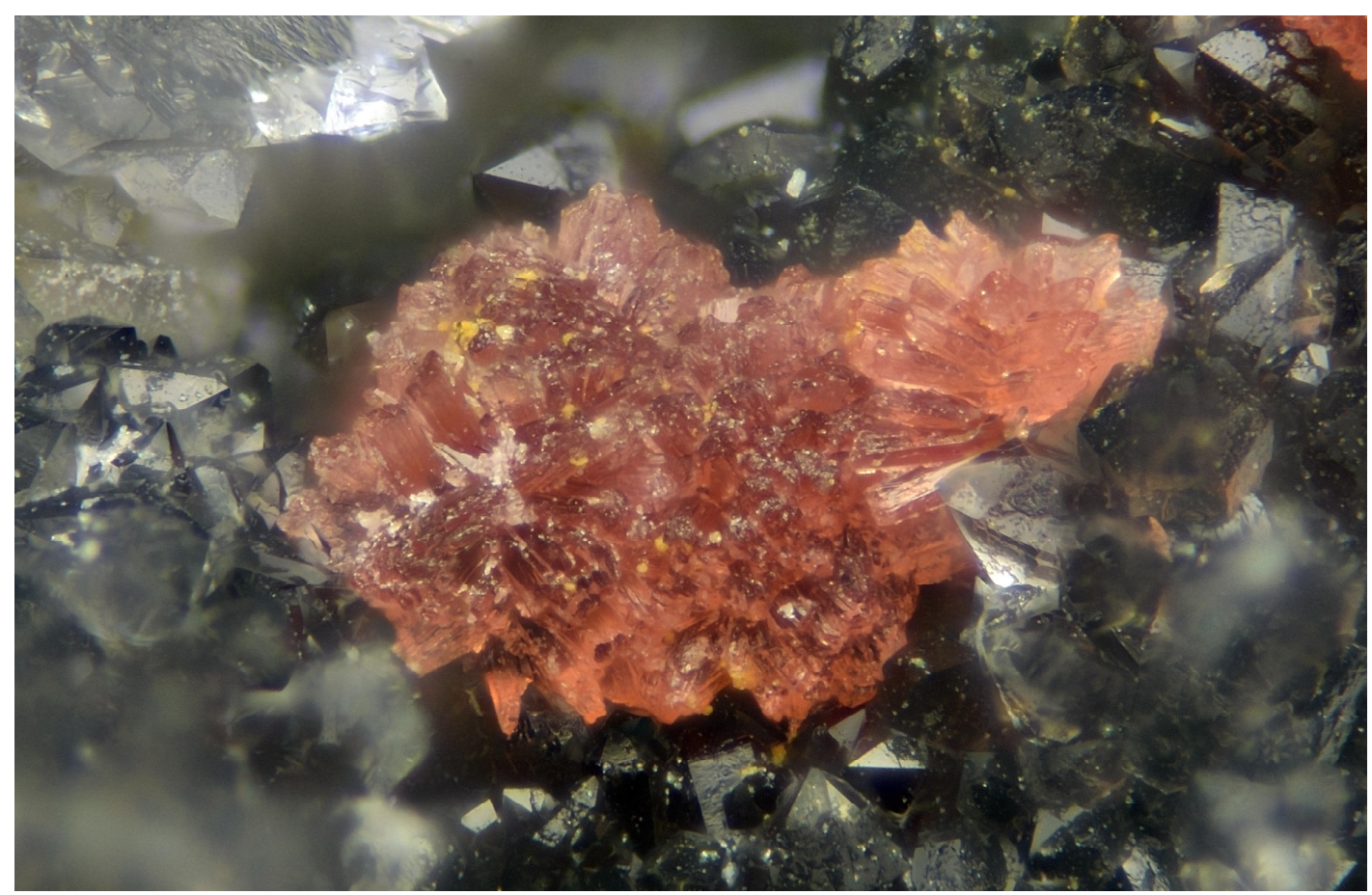

Figure 9 : Érythrite de Petite-Hoursinne. L = 0,71 mm. Coll. S. Puccio, Photo P. Chollet. 


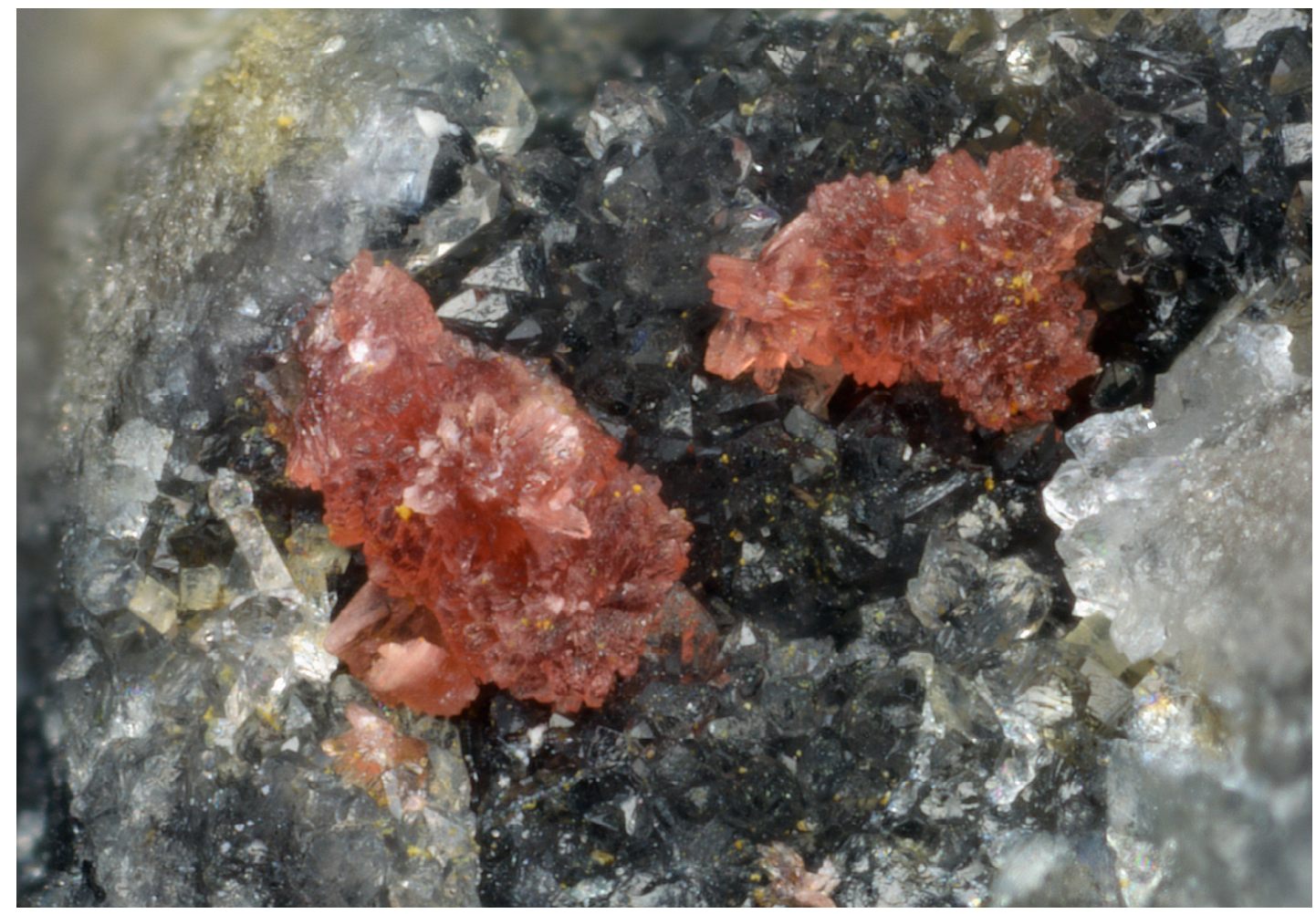

Figure 10 : Érythrite de Petite-Hoursinne. $\mathrm{L}=1,50 \mathrm{~mm}$. Coll. S. Puccio, Photo P. Chollet

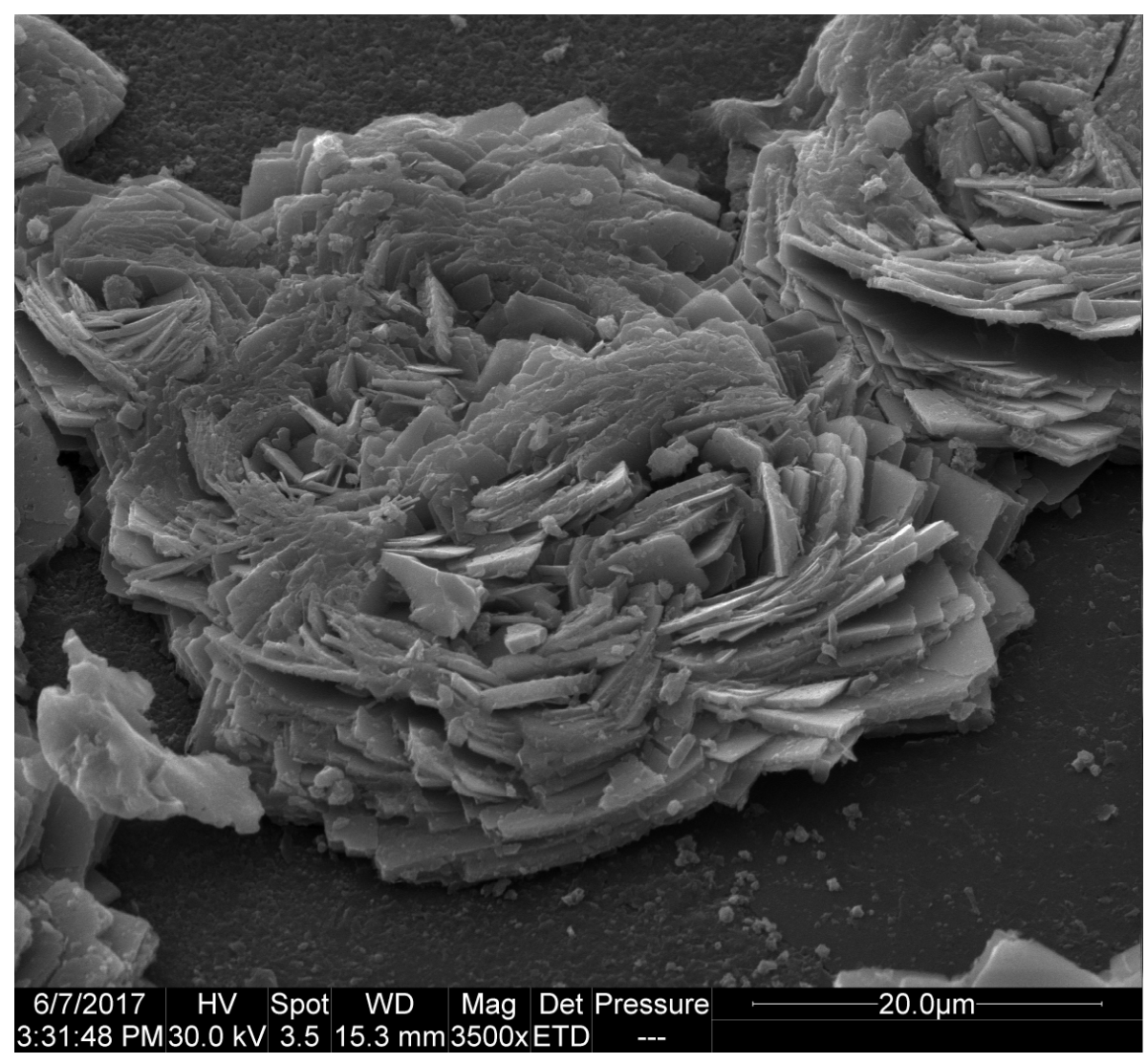

Figure11 : Érythrite de Petite-Hoursinne. Échantillon OUR-2, photographies au microscope électronique à balayage, mode électrons secondaires. 


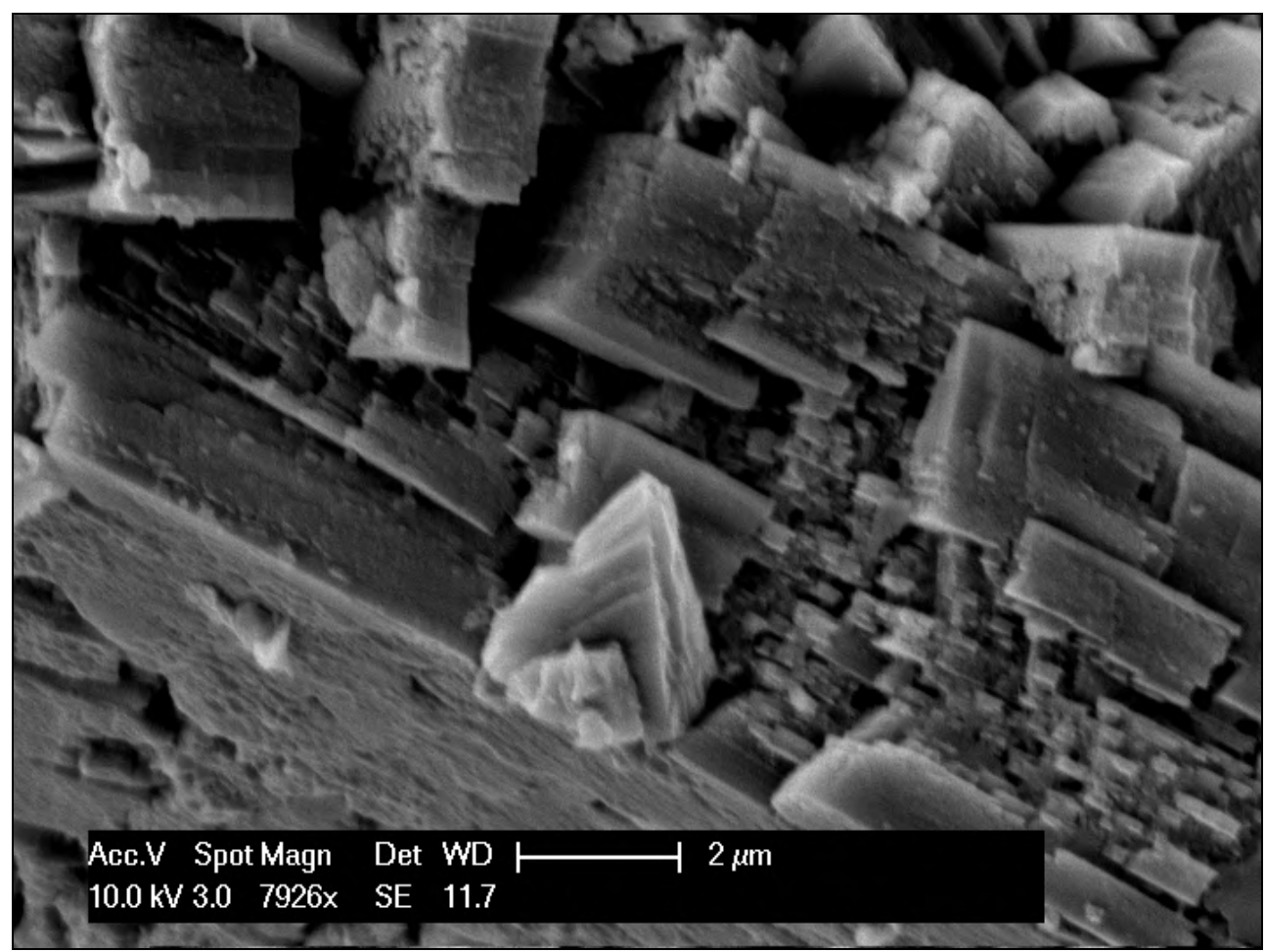

Figure 12 : Cristal d'érythrite de Petite-Hoursinne. Echantillon OUR-4c, photographies au microscope électronique à balayage, mode électrons secondaires.

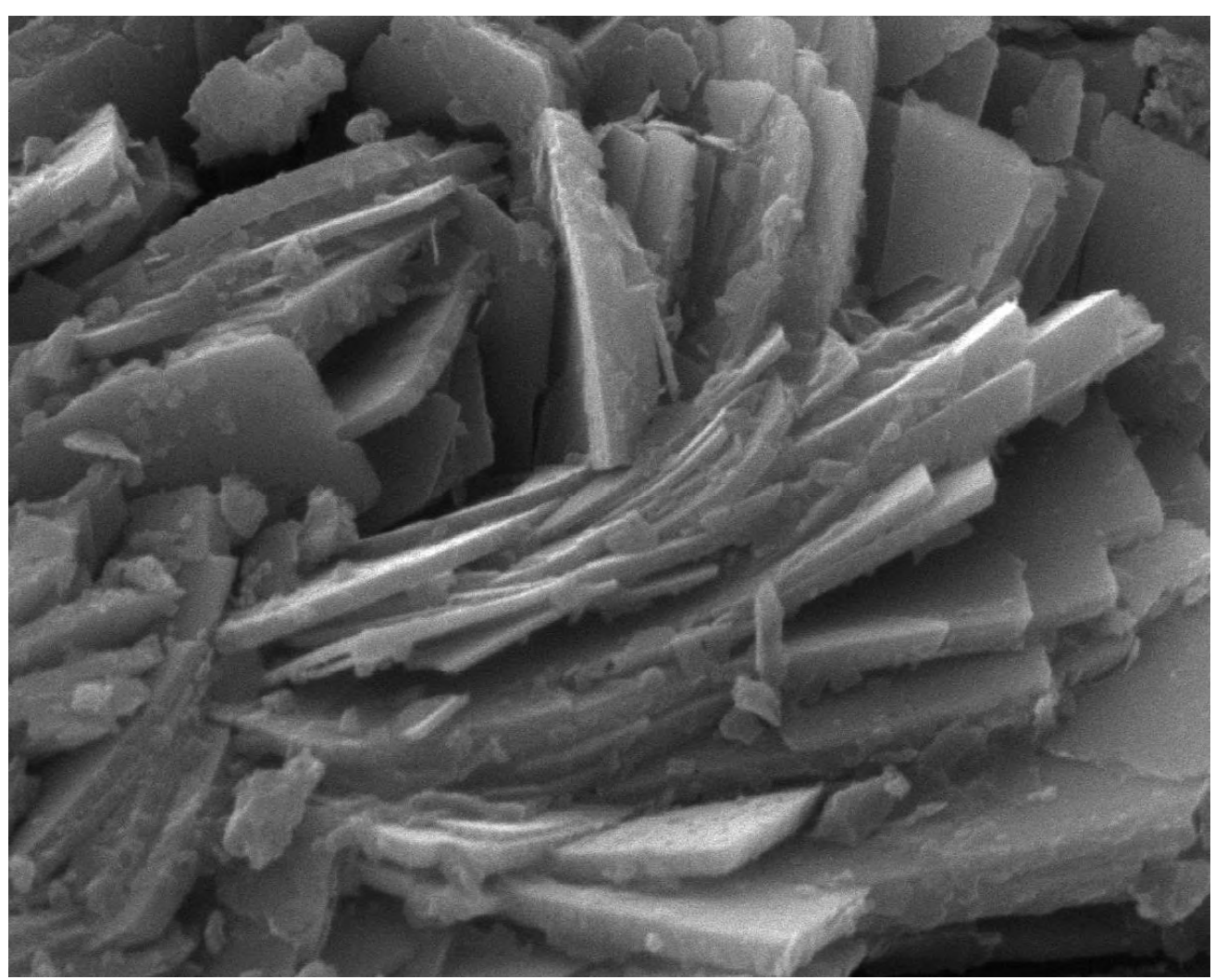

Figure 13 : Érythrite de Petite-Hoursinne. Echantillon OUR-02, photographies au microscope électronique à balayage, mode électrons secondaires. $\mathrm{L}=100 \mu \mathrm{m}$. 


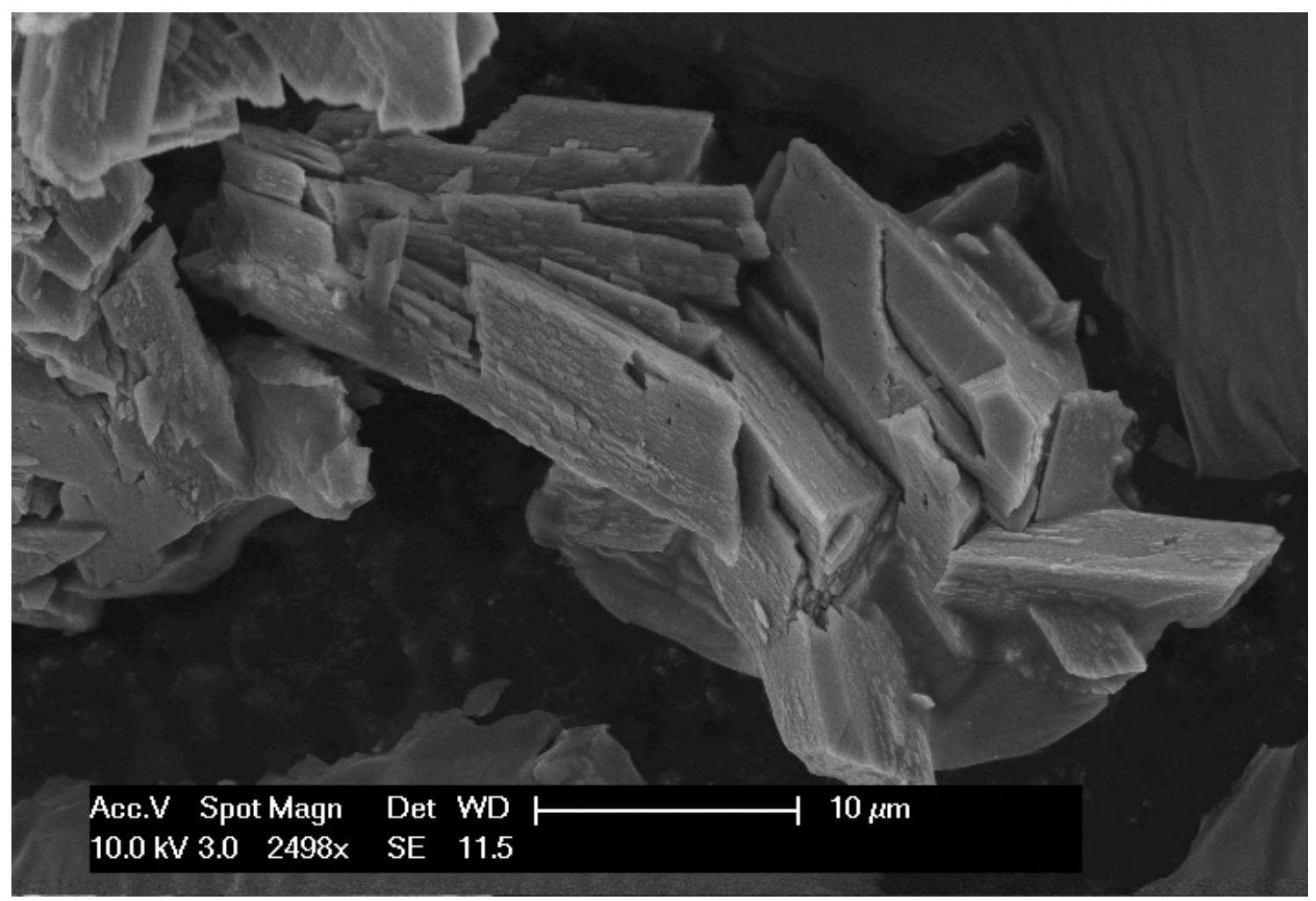

Fig. 14 : Erythrite de Petite-Hoursinne. Echantillon OUR-4d-2, photographies au microscope électronique à balayage, mode électrons secondaires.

\subsection{Galène, $\mathrm{PbS}$}

La galène, identifiée visuellement, est fréquente en petits grains dans les haldes. On peut également l'observer au fond de la galerie en petits cubes corrodés dans de minces joints de quartz d'une épaisseur de quelques millimètres à un centimètre seulement. Il ne s'agit pas vraiment d'un filon mais plutôt d'un chapelet de petits cristaux de galène pouvant, d'après nos observations, atteindre un centimètre d'arête mais dans ce type de gîte ardennais il n'aurait pas été étonnant d'y trouver localement des nids de gros cristaux pluri-centimétriques comme ce fut le cas notamment à Masbourg (Blondieau et al., 2017) ou encore à Tellin. Les cristaux d'Hoursinne se présentent en cubes avec de petites faces de l'octaèdre $\{111\}$ (Figure 15). Ils sont fréquemment emballés par des cristaux de quartz dans lesquels s'observent aussi des inclusions gris acier qui, comme à Masbourg (Blondieau et al., 2017), sont sans doute des "gouttelettes" de galène.

La galène est également observée en injections dans la roche bleutée très compacte ou localement "limoniteuse". Elle minéralise parfois aussi les coquilles de fossiles brachiopodes ; observation déjà signalée par Lohest (1900) : "Dans ces roches, la galène remplace souvent le test calcaire de grands brachiopodes, comme en témoignent les échantillons que j'ai recueillis et qui proviennent d'un filon situé au bord du ruisseau ..."

À l'intérieur de ces coquilles ou sur les moules internes des fossiles, la galène peut parfois se développer en petits cristaux associés à la dolomite, au quartz et, plus rarement, à l'érythrite. 
Des analyses par spectrométrie de fluorescence $\mathrm{X}$ de cette galène ne montrent pas d'anomalies chimiques particulières par rapport aux autres galènes belges, à part des teneurs en antimoine et en argent un peu au-dessus des valeurs moyennes. Cette corrélation positive entre antimoine et argent a déjà été constatée dans des sphalérites rubanées ("schalenblendes") dont l'étude est en cours.

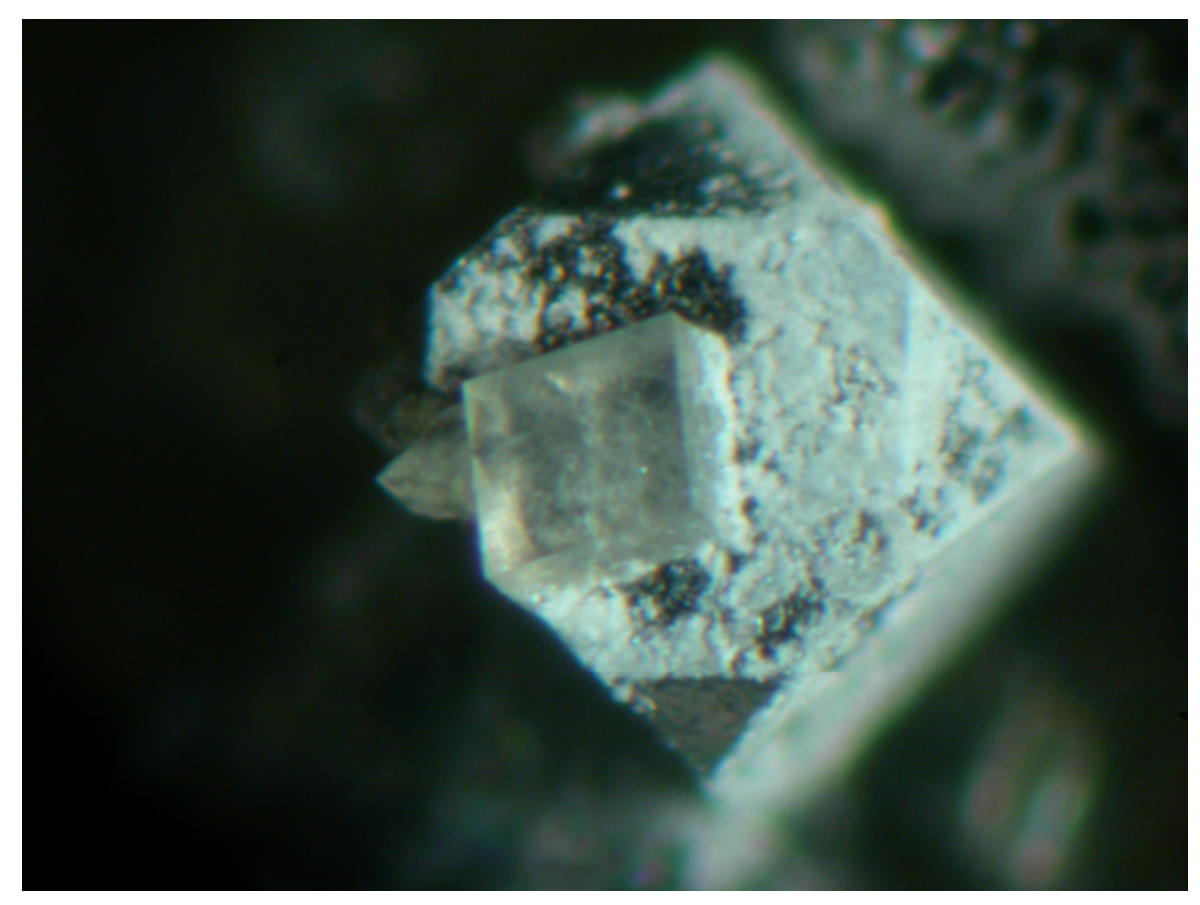

Figure 15 : Galène de Petite-Hoursinne. Cristal corrodé en surface avec facettes $\{111\}$ surmonté d'un cristal de dolomite. $\mathrm{L}=2 \mathrm{~mm}$. Photo $\mathrm{M}$. Blondieau.

\subsection{Goethite/limonite, $\mathrm{FeO}(\mathrm{OH})$}

La goethite est parfois observée en jolie croûte multicolore sur des cristaux de galène avec cérusite et quartz (Figure 16). Elle est également un des principaux constituants des parties brun rouille des roches "limoniteuses" où elle se trouve associée à des oxydes de manganèse généralement amorphes et non identifiables.

Signalons également une analyse par diffraction des rayons $\mathrm{X}$ de cristaux identifiés visuellement comme de la pyrite probable et qui a montré qu'ils étaient entièrement transformés en goethite. 


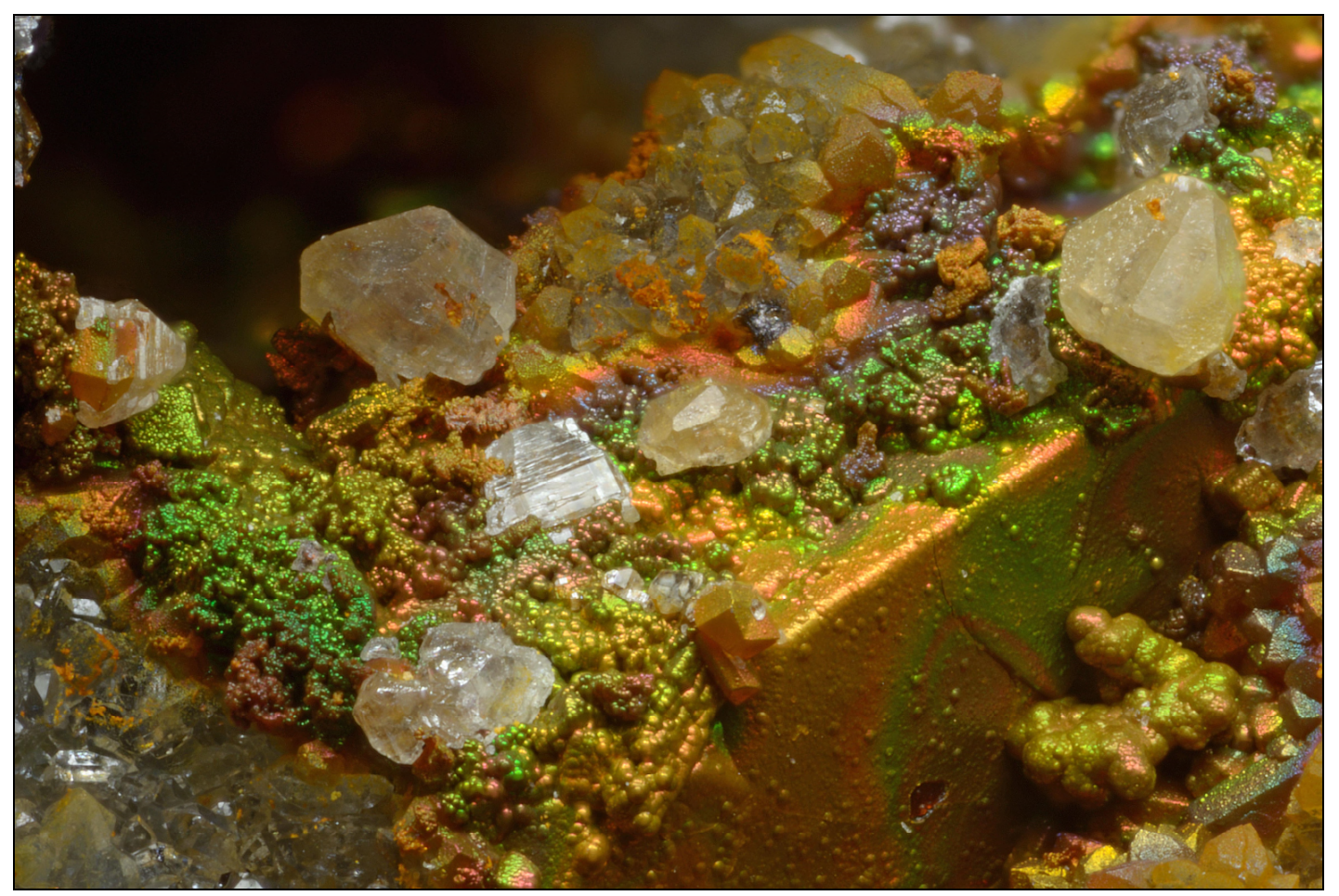

Figure 16 : Goethite irisée en croûte sur un cube de galène avec cristaux de cérusite en macles pseudo-hexagonale et quartz, Petite-Hoursinne. $\mathrm{L}=2,4 \mathrm{~mm}$. Photos P.Chollet.

\subsection{Kaolinite, $\mathrm{Al}_{4}\left[\mathrm{Si}_{4} \mathrm{O}_{10}\right](\mathrm{OH}) 8$}

La kaolinite, identifiée par diffraction des rayons X, se présente en fins dépôts pulvérulents blanc sur le quartz, la dolomite et la galène. Dans les rares blocs contenant les minéraux de cobalt, la kaolinite est parfois observée colorée en rose. La kaolinite est un minéral typique des faciès d'altération.

\subsection{Malachite, $\mathrm{Cu}_{2}\left(\mathrm{CO}_{3}\right)(\mathrm{OH})_{2}$}

La malachite a été observée en enduits verts et en minuscules cristaux mal formés au voisinage de plages de chalcopyrite. Son identification visuelle est confirmée par diffraction des rayons $\mathrm{X}$.

\subsection{Manganite, $\mathrm{Mn}^{3+} \mathrm{O}(\mathrm{OH})$ et pyrolusite, $\mathrm{MnO}_{2}$}

Des analyses chimiques qualitatives par EDS sur de minuscules cristaux noirs indiquent la seule présence des éléments $\mathrm{Mn}$ et $\mathrm{O}$. Les photos en microscopie électronique (Figure 17) montrent des cristaux très bien formés de quelques dizaines de microns de longueur. Ils sont associés en fagots et ont une morphologie pouvant faire penser à celle de la manganite. Des analyses par diffraction des rayons $\mathrm{X}$ mettent en évidence une association de manganite et de pyrolusite (Tableau 2). La manganite est, en effet, un minéral qui se transforme facilement en pyrolusite par épigénie (déshydratation et oxydation du manganèse de l'état +3 à son état d'oxydation maximal +4 ). La coexistence des raies de diffraction des deux espèces indique 
que les cristaux de Petite-Hoursinne sont de la manganite partiellement épigénisée en pyrolusite.

Tableau 2 : Diffractogramme de poudres de rayons X de la manganite de Petite Hoursinne (Diffractomètre à 4 cercles Rigaku Xcalibur, détecteur EOS, radiation MoK $\alpha$ ), comparé à ceux de la manganite d'Allemagne et de la pyrolusite de synthèse.

\begin{tabular}{|c|c|c|c|c|}
\hline \multirow{2}{*}{$\begin{array}{c}\text { Minéral de } \\
\text { Petite-Hoursinne }\end{array}$} & \multicolumn{2}{|c|}{$\begin{array}{l}\text { Manganite } \\
\text { Allemagne }\end{array}$} & \multicolumn{2}{|c|}{$\begin{array}{l}\text { Pyrolusite** } \\
\text { de synthèse }\end{array}$} \\
\hline & $d(\AA)$ & $\mathrm{I} / \mathrm{I}_{0}(\%)$ & $d(\AA)$ & $\mathrm{I} / \mathrm{I}_{0}(\%)$ \\
\hline $3,401(\mathrm{TF})$ & 3,405 & 100 & - & - \\
\hline 3,124 (TF) & - & - & 3,11 & 100 \\
\hline $2,640(\mathrm{~m})$ & 2,639 & 24 & - & - \\
\hline 2,517 (f) & 2,524 & 12 & - & - \\
\hline 2,407 (TF) & $\begin{array}{l}2,417 \\
2,414\end{array}$ & $\begin{array}{l}17 \\
16\end{array}$ & 2,407 & 55 \\
\hline 2,261 (f) & 2,271 & 10 & - & - \\
\hline $2,194(\mathrm{~m})$ & 2,196 & 9 & 2,199 & 8 \\
\hline 2,121 (f) & - & - & 2,110 & 16 \\
\hline 1,974 (f) & - & - & 1,9681 & 5 \\
\hline $1,780(\mathrm{~m})$ & 1,782 & 21 & - & - \\
\hline 1,701 (Tf) & 1,703 & 10 & - & - \\
\hline $1,667(\mathrm{~m})$ & $\begin{array}{l}1,672 \\
1,670\end{array}$ & $\begin{array}{l}17 \\
16\end{array}$ & - & - \\
\hline $1,627(\mathrm{~F})$ & 1,636 & 9 & 1,6234 & 55 \\
\hline $1,561(\mathrm{~m})$ & - & - & 1,5554 & 14 \\
\hline $1,499(\mathrm{~m})$ & 1,500 & 10 & - & - \\
\hline $1,427(\mathrm{~m})$ & $\begin{array}{l}1,437 \\
1,430\end{array}$ & $\begin{array}{l}5 \\
5\end{array}$ & 1,437 & 8 \\
\hline & 1,430 & $\begin{array}{l}5 \\
-\end{array}$ & & \\
\hline $\begin{array}{c}1,404(\mathrm{~m}) \\
-\end{array}$ & $\begin{array}{c}- \\
1,324\end{array}$ & $\overline{9}$ & $\begin{array}{c}1,3912 \\
-\end{array}$ & $\begin{array}{l}8 \\
-\end{array}$ \\
\hline $1,317(\mathrm{~m})$ & 1,319 & 5 & - & - \\
\hline $1,308(\mathrm{~m})$ & - & - & $\begin{array}{l}1,3064 \\
1,3045\end{array}$ & $\begin{array}{l}20 \\
20\end{array}$ \\
\hline 1,262 (Tf) & 1,262 & 4 & - & - \\
\hline- & 1,244 & 3 & - & - \\
\hline 1,206 (f) & - & - & 1,2029 & 3 \\
\hline 1,180 (f) & 1,180 & 6 & - & - \\
\hline 1,158 (f) & 1,158 & 5 & - & - \\
\hline 1,134 (f) & 1,135 & 7 & - & - \\
\hline- & - & - & 1,1232 & 5 \\
\hline- & 1,114 & 2 & - & - \\
\hline 1,097 (f) & 1,098 & 4 & 1,1000 & 2 \\
\hline 1,078 (f) & 1,079 & 4 & - & - \\
\hline
\end{tabular}

* Fiche ICDD 41-1379 : Manganite de Harz Mountains, Allemagne.

** Fiche ICDD 24-735 : Pyrolusite de synthèse

$(\mathrm{TF})$ : Très fort, $(\mathrm{F})$ : fort, $(\mathrm{m})$ : moyen, $(\mathrm{f})$ : faible, $(\mathrm{Tf})$ : très faible. 

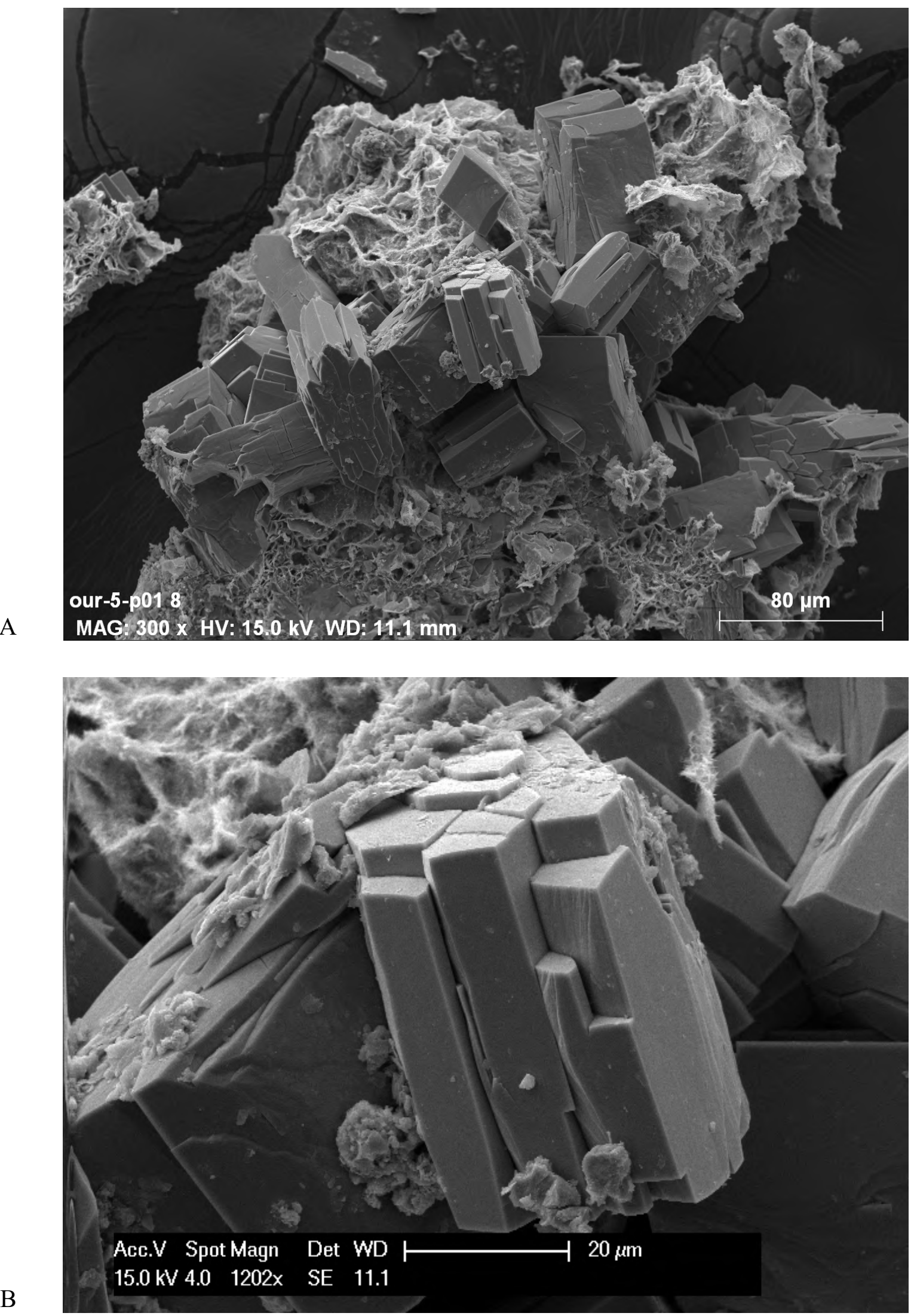

Figure17 : Manganite partiellement épigénisée en pyrolusite de Petite Hoursinne. Échantillon OUR-5, photographies au microscope électronique à balayage, mode électrons secondaires. 


\subsection{Mimétite, $\mathrm{Pb}_{5}\left(\mathrm{AsO}_{4}\right)_{3} \mathrm{Cl}$}

La mimétite de Petite-Hoursinne se présente le plus souvent en aiguilles blanches (Figures $18 \mathrm{~A}$ et $18 \mathrm{~B}$ ) ou en petits barillets hexagonaux ; les cristaux les plus épais prennent fréquemment une couleur jaune or (Figure 18C). Les analyses par diffraction des rayons X ont mis en évidence qu'il s'agissait d'un membre de la série pyromorphite-mimétite, et les analyses chimiques qualitatives par EDS sur l'échantillon OUR-1 de couleur jaune, ont montré la présence des éléments majoritaires $\mathrm{Pb}$, As et $\mathrm{O}$. L'absence de phosphore indique qu'il s'agit bien de mimétite et non de pyromorphite. Généralement, les minéraux de ce groupe sont souvent des membres intermédiaires, ce qui ne semble pas être le cas à Hoursinne où le minéral analysé correspond au pôle pur mimétite.

A

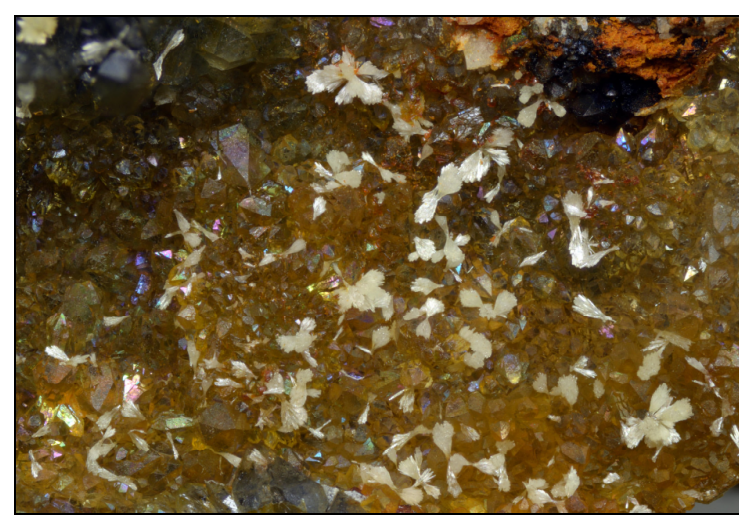

$\mathrm{B}$

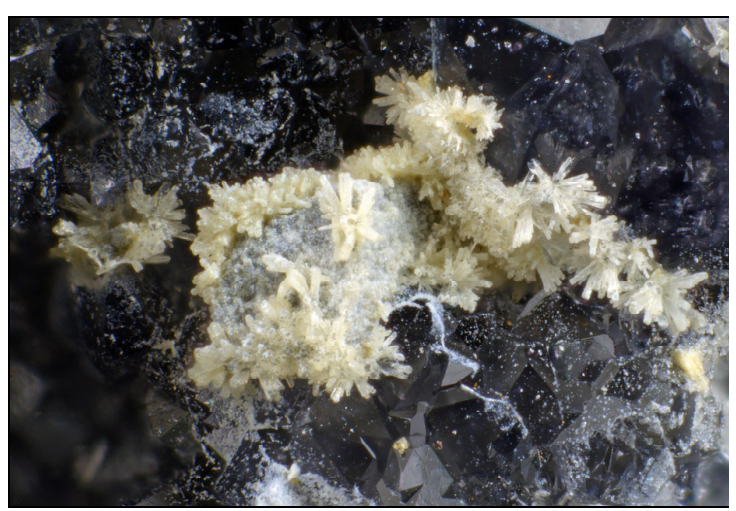

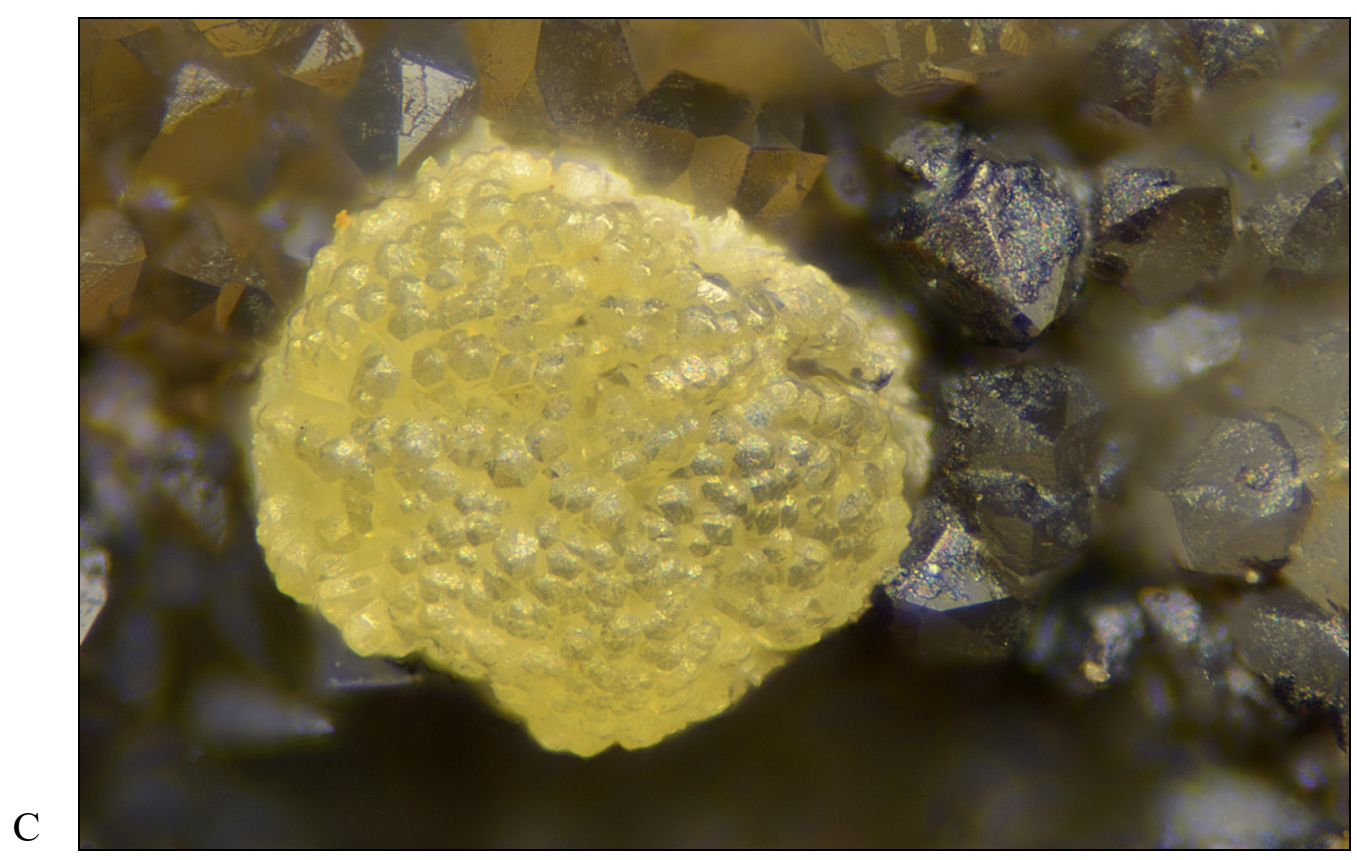

Figure 18 : Mimétite de Petite-Hoursinne. (A) Petites gerbes de cristaux blancs sur quartz. $\mathrm{L}=3,50 \mathrm{~mm}$. (B) Cristaux blancs sur galène $\mathrm{L}=1,20 \mathrm{~mm}(\mathrm{C})$ Agrégat jaune sur quartz. $\mathrm{L}=0,55 \mathrm{~mm}$. Photos $\mathrm{P}$. Chollet. 
La mimétite, assez rare à Petite-Hoursinne, est observée en association avec la galène et la cérusite dans des cavités tapissées par du quartz microcristallin de la roche gréseuse limoniteuse. En Belgique, la mimétite n'est pas une espèce fréquente mais elle a déjà été recensée dans trois localités ardennaises : Vielsalm dans l'Ordovicien (Hatert et al., 1998), Hourt dans le Cambrien (Lefèvre \& Hatert, 2003), et La Roche-en-Ardenne (Blondieau \& Hatert, 2009) dans le Dévonien (Formation de Villé, comme à Hoursinne).

\subsection{Pyrite, $\mathrm{FeS}_{2}$}

De très minces et rares joints lardant la roche sont identifiés visuellement comme étant de la pyrite, ce qui est confirmé par diffraction des rayons X. Il en est de même de minuscules cristaux cubo-octaédriques.

\subsection{Quartz, $\mathrm{SiO}_{2}$}

Le quartz est abondant dans la roche qui encaisse les minéralisations. Il se présente en plages constituées de nombreux petits cristaux millimétriques serrés les uns contre les autres. Le minéral est peu spectaculaire et souvent teinté par des enduits bruns ou noirs. Son identification est réalisée visuellement. En couronne, autour des cristaux de galène, on remarque fréquemment des agrégats de cristaux de quartz de seconde génération; parfois même, ils emballent totalement la galène. Ces cristaux de quelques millimètres de longueur présentent alors un habitus cristallin avec des faces prismatiques et pyramidales. Ils sont incolores ou grisâtres avec parfois des inclusions en gouttelettes à l'éclat métallique ; sans doute de la galène.

\subsection{Soufre, $S$}

Un cristal jaune pâle de soufre en bipyramide aigüe a été observé dans une petite géode avec galène. L'identification du minéral est confirmée par diffraction des rayons $\mathrm{X}$.

La présence de soufre sur la galène a déjà été signalée, notamment à La Roche-en-Ardenne (Blondieau \& Hatert, 2009) et à Masbourg (Blondieau et al., 2017). Il s'agit d'un minéral secondaire produit par altération des sulfures dans les haldes sous l'action des agents météoriques.

\subsection{Sphalérite, $(\mathrm{Zn}, \mathrm{Fe}) \mathrm{S}$}

La sphalérite se présente avec galène et chalcopyrite en petites esquilles jaune miel à orange et parfois en petits cristaux brun corrodés sur des cristaux de quartz. Le minéral est peu fréquent à Petite-Hoursinne et est identifié par diffraction des rayons X. Parfois, on l'observe dans la paragenèse des petites boules roses d'érythrite, ce qui nous avait conduit aussi à penser à la présence de köttigite.

\subsection{Todorokite, (Na,Ca,K,Ba,Sr) $)_{1-x}(\mathrm{Mn}, \mathrm{Mg}, \mathrm{Al})_{6} \mathrm{O}_{12.3-4 \mathrm{H}_{2} \mathrm{O}}$}

Un minéral en feuillets bruns violacés sur dolomite très altérée et "limonite" est identifié par diffraction des rayons $\mathrm{X}$ comme de la todorokite. Sous cet aspect, il ressemble beaucoup à la 
todorokite de La Roche-en-Ardenne (Blondieau \& Hatert, 2009) et de Masbourg (Blondieau et al., 2017).

\section{Discussion}

Les arséniates ne sont pas fréquents à Petite-Hoursinne, et il est probable qu'ils ne se sont développés que dans quelques parties très localisées du gîte. Deux analyses géochimiques sur sédiments du ruisseau d'Hoursinne, à proximité de la galerie de Petite-Hoursinne, l'un à quelques 600 mètres en amont (analyse $\mathrm{n}^{\circ} 1550241$ ) et l'autre à environ $300 \mathrm{~m}$ en aval (analyse $\mathrm{N}^{\circ} 1550239$ ), n'ont pas mis en évidence d'anomalie particulière ni en arsenic ni en cobalt (Sonnet, 2017 ; localisations sur le médaillon en Figure 1).

Le mode de gisement à La Roche-en-Ardenne est similaire à celui de Petite-Hoursinne, car les échantillons avec mimétite et érythrite n'avaient été observés que très localement en place, dans un très petit affleurement le long de la route, et nous n'en avions pas revu dans l'important matériel bien minéralisé qui avait été enlevé lors de la sécurisation du talus. Les éléments rares As, Co et Ni, qui s'expriment dans des minéralisations à Petite-Hoursinne mais aussi à La Roche-en-Ardenne, sont probablement liées à la géochimie particulière de la formation de Villé, une formation du Dévonien inférieur dans laquelle se trouvent de nombreuses traces de galène (Léon Dejonghe, comm. pers., 2009).

Nous pouvons aussi citer Masbourg (Blondieau et al, 2017), ainsi que le plus gros filon plombifère ardennais sur le territoire belge, Longvilly, qui sont tous les deux logés, au moins en partie, dans la formation de Villé. À propos de Longvilly, Legrand (1970) écrit : "A l'ouest, le filon donne des ramifications diffuses dans les quartzophyllades du Siegenien moyen, s'écartant jusqu'à plusieurs mètres du filon principal, ayant donné lieu à des "chassages" fructueux" ; le Siegenien moyen ("S2") signalé par Legrand (1970) correspond bien à la formation de Villé (Tableau 1). Sur ses cartes géologiques, X. Stainier localise également quelques autres petits indices plombifères aujourd'hui inaccessibles, notamment aux Amonines (Stainier, 1898) au sud d'Erezée, et à Laneuville-aux-bois au sud-ouest de Tenneville (Stainier, 1900). Il faut également citer une anomalie plombo-zincifère à Borzée (Martin et al., 1990).

Le Tableau 3 ci-dessous reprend la liste des espèces minérales observées à Petite-Hoursinne. 
Tableau 3 : Espèces minérales recensées à Petite-Hoursinne.

\begin{tabular}{|c|c|}
\hline Minéral & Formule chimique idéale \\
\hline Anglésite & $\mathrm{PbSO}_{4}$ \\
\hline Aragonite & $\mathrm{CaCO}_{3}$ \\
\hline Calcite & $\mathrm{CaCO}_{3}$ \\
\hline Cérusite & $\mathrm{PbCO}_{3}$ \\
\hline Chalcopyrite & $\mathrm{CuFeS}_{2}$ \\
\hline Covelline & $\mathrm{CuS}$ \\
\hline Dolomite & $\mathrm{CaMg}\left(\mathrm{CO}_{3}\right)_{2}$ \\
\hline Erythrite & $\mathrm{Co}_{3}\left(\mathrm{AsO}_{4}\right)_{2} .8 \mathrm{H}_{2} \mathrm{O}$ \\
\hline Galène & $\mathrm{PbS}$ \\
\hline Goethite & $\mathrm{FeO}(\mathrm{OH})$ \\
\hline Kaolinite & $\mathrm{Al}_{4}\left[\mathrm{Si}_{4} \mathrm{O}_{10}\right](\mathrm{OH})_{8}$ \\
\hline Malachite & $\mathrm{Cu}_{2}\left(\mathrm{CO}_{3}\right)(\mathrm{OH})_{2}$ \\
\hline Manganite & $\mathrm{Mn}^{3+} \mathrm{O}(\mathrm{OH})$ \\
\hline Mimétite & $\mathrm{Pb}_{5}\left(\mathrm{AsO}_{4}\right)_{3} \mathrm{Cl}$ \\
\hline Pyrite & $\mathrm{FeS}_{2}$ \\
\hline Pyrolusite & $\mathrm{MnO}_{2}$ \\
\hline Quartz & $\mathrm{SiO}_{2}$ \\
\hline Soufre & $\mathrm{S}$ \\
\hline Sphalérite & $(\mathrm{Zn}, \mathrm{Fe}) \mathrm{S}$ \\
\hline Todorokite & $\begin{array}{c}(\mathrm{Na}, \mathrm{Ca}, \mathrm{K}, \mathrm{Ba}, \mathrm{Sr})_{1-\mathrm{x}} \\
(\mathrm{Mn}, \mathrm{Mg}, \mathrm{Al})_{6} \mathrm{O}_{12} .3-4 \mathrm{H}_{2} \mathrm{O}\end{array}$ \\
\hline
\end{tabular}

\section{Conclusions}

Le petit gite plombifère de Petite-Hoursinne présente une minéralogie intéressante avec érythrite et mimétite, deux arséniates rares en Belgique. L'érythrite n'était d'ailleurs recensée qu'à La Roche-en Ardenne, un gisement qui se trouve dans la même formation géologique que celle de Petite-Hoursinne : la Formation de Villé. Petite Hoursinne est donc la seconde occurrence belge de ce minéral.

\section{Remerciements :}

Nous souhaitons remercier Pascal Chollet qui a réalisé les magnifiques photos de nos très petits minéraux ainsi que Philippe Compère pour son aide lors de l'utilisation du microscope électronique à balayage. 


\section{Références}

Asselberghs E. (1946). L'éodévonien de l'Ardenne et des régions voisines. Mémoire de l'Institut de Géologie de l'Université de Louvain, 16, 598 pages.

Blondieau M. (1993). Les ressources minérales de la Calestienne et leur exploitation. De la Meuse à l'Ardenne, 16, 35-53.

Blondieau M. \& Hatert F. (2009). Minéralogie de l'ancienne mine de plomb de La Roche-enArdenne (Belgique). Le Règne Minéral, 88, 5-20.

Blondieau M., Puccio S. \& Hatert, F. (2017). Minéralogie de l'ancienne mine de plomb de Masbourg (Nassogne, Province de Luxembourg, Belgique). Bulletin de la Société Royale des Sciences de Liège, 86, 123-155.

Hatert F., Blondieau M., Dehove J. \& Fransolet A.M (1998). Les minéraux secondaires en relation avec les sulfures de la région de Vielsalm. Bulletin de la Société royale des Sciences de Liège, 67 (6), 281.

Lefèvre P. \& Hatert F. (2003). Or natif, arsénopyrite et minéraux secondaires dans les quartzites devilliens de Hourt, Massif de Stavelot, Belgique. Geologica Belgica, 6/34, 193-200.

Legrand R. (1970). L'ancienne mine de Longvilly, Service géologique de Belgique, Prof. Paper, 13 , 7 pages.

Lohest M. (1900). Filons de galène de Harre - Annales de la Société géologique de Belgique, 28, B.51

Marion J.M. \& Barchy L. (2007). Description de l'affleurement n5520748, commune d'Erezée. Carte géologique de Wallonie.

Martin H., Bolle J.N., Brodkom F., Buvé L., Michiels D., Pingot J.L. \& Sondag F. (1990). Valorisation de l'inventaire géochimique des ressources métallifères de la Wallonie; Rapport final : synthèse des résultats. Ministère de la Région Wallonne, service Ressources du Sous-sol, 54 pages.

Pirotte F. (1966). L'Industrie métallurgique de la Terre de Durbuy de 1480 à 1625. Ses rapports avec la métallurgie liégeoise, Bulletin de l'Institut archéologique liégeois, Tome LXXIV, 145-210.

Sonnet P. (2017). Inventaire géochimique des ressources métallifères de la Wallonie. http://geologie.wallonie.be/home/thematiques-sous-sol/inventaire-geochimique.html (accès juin 2017)

Stainier X. (1898). Carte géologique (1:40000) N¹78 (planchettes 5-6 de la feuille LV de la carte topographique), Hotton-Dochamps, institut cartographique militaire.

Stainier X. (1900). Carte géologique (1:40000) N¹96 (planchettes 5-6 de la feuille LX de la carte topographique), Amberloup-Flamierge, institut cartographique militaire.

Stainier X. (1902). Carte géologique (1:40000) N¹69 (planchettes 1-2 de la feuille LV de la carte topographique), Durbuy-Mormont, institut cartographique militaire. 